\title{
A manifold calculus approach to link maps and the linking number
}

\author{
BRIAN A MUNSON
}

\begin{abstract}
We study the space of link maps $\operatorname{Link}\left(P_{1}, \ldots, P_{k} ; N\right)$, the space of smooth maps $P_{1} \sqcup \cdots \sqcup P_{k} \rightarrow N$ such that the images of the $P_{i}$ are pairwise disjoint. We apply the manifold calculus of functors developed by Goodwillie and Weiss to study the difference between it and its linear and quadratic approximations. We identify an appropriate generalization of the linking number as the geometric object which measures the difference between the space of link maps and its linear approximation. Our analysis of the difference between link maps and its quadratic approximation resembles recent work of the author on embeddings, and is used to show that the Borromean rings are linked.
\end{abstract}

57Q45, 57R99; 55P99, 57M25

\section{Introduction}

Let $P_{1}, \ldots, P_{k}$ be smooth closed manifolds of dimensions $p_{1}, \ldots, p_{k}$, and let $N$ be a smooth manifold.

Definition 1.1 The space of link maps of $P_{1}, \ldots, P_{k}$ in $N$ is

$$
\operatorname{Link}\left(P_{1}, \ldots, P_{k} ; N\right)=\left\{f_{i}: P_{i} \rightarrow N \mid f_{i}\left(P_{i}\right) \cap f_{j}\left(P_{j}\right)=\varnothing \text { for } i \neq j\right\},
$$

topologized as a subspace of the space of smooth maps.

It is important to distinguish this from the space of embeddings of the disjoint union, since all we assume about the individual maps $f_{i}$ is that they are smooth. Thus we are ignoring "self-linking". Alternately, one could study the case where the $f_{i}$ are immersions, a case considered by Hatcher and Quinn [10] and Schneiderman and Teichner [23]. Our description of the linking number was inspired by Section 1.4 of Goodwillie, Klein and Weiss [4], which describes double point obstructions to embeddings, and we also rely heavily on [22] for technical details, in which the author measured secondary obstructions (to the double point obstruction) for embedding a manifold in Euclidean space. 
The study of the space of link maps began with the work of Milnor [20], who worked in the classical case where the $P_{i}$ are circles and $N$ is a 3-manifold. He was mostly concerned with the fundamental group of the complement of a given link map, which in this case may be assumed to be embedded. This was further investigated by Levine [18], Habegger and Lin [9] and many others. The higher dimensional analog, which we are most concerned with here, has been extensively studied by Hatcher and Quinn [10], Koschorke [16; 15; 14; 13], Koschorke and Rolfsen [17], Habegger and Kaiser [8], Massey and Rolfsen [19], Scott [24], Skopenkov [25], Schneiderman and Teichner [23] and Klein and Williams [12].

Our analysis of "linking manifolds" in Section 5 owes much to the work of Hatcher and Quinn [10], as well as to the survey paper of Goodwillie, Klein, and Weiss [5]. It also intersects the recent work of Klein and Williams [12, Section 9]. Our discussion of the linking number also relates to work of Chernov and Rudyak [1]. But for us, a linking number depends on the choice of a path between the link in question and the unlink, while they wish to keep track of such choices.

Our goal is to understand the space of link maps from the point of view of the manifold calculus of functors developed by Weiss and Goodwillie [26; 7]. We consider the space of link maps $\operatorname{Link}\left(P_{1}, \ldots, P_{k} ; N\right)$ as one value of a contravariant functor from the poset of open subsets of $P_{1} \sqcup \cdots \sqcup P_{k}$. We describe briefly in Section 4 how this theory assigns $j$-th degree "polynomial" approximations to such a functor $F$, denoted $\mathcal{T}_{j} F$. We will give a geometric description of the fiber of $\operatorname{Link}\left(P_{1}, \ldots, P_{k} ; N\right) \rightarrow$ $\mathcal{T}_{j} \operatorname{Link}\left(P_{1}, \ldots, P_{k} ; N\right)$ when $j=1,2$. This geometric description is in terms of cobordism spaces, the relevant details of which can be found in [22], and the relevant definitions and results are discussed in Section 3. Our Theorem 1.2 (resp. Theorem 1.3) states that there is a map from the homotopy fiber of the space of link maps to its linear (resp. quadratic) approximation to a cobordism space. As the difference between the space of link maps and its linear (resp. quadratic) approximation begins with quadratic (resp. cubic) information, the relevant cobordism spaces are models for the quadratic (resp. cubic) homogeneous parts of the Taylor tower for these functors (see Section 4 for explanation of this terminology). The real content of these theorems is that the maps in question have geometric content, and one of them has a connectivity that the author and Tom Goodwillie [6] have computed, and will appear soon. Choose a basepoint $\left(f_{1}, f_{2}\right) \in \operatorname{Link}\left(P_{1}, P_{2} ; N\right)$.

\section{Theorem 1.2 There is a map}

$l_{2}: \operatorname{hofiber}\left(\operatorname{Link}\left(P_{1}, P_{2} ; N\right) \rightarrow \operatorname{map}\left(P_{1}, N\right) \times \operatorname{map}\left(P_{2}, N\right)\right) \rightarrow \Omega C_{2}\left(P_{1}, P_{2} ; N\right)$,

which can be interpreted as the "generalized linking number". 
The space $C_{2}\left(P_{1}, P_{2} ; N\right)$ is a representing space for the cobordism, twisted by a bundle described in Section 5, of

$$
E_{12}=\operatorname{holim}\left(P_{1} \stackrel{f_{1}}{\rightarrow} N \stackrel{f_{2}}{\leftarrow} P_{2}\right),
$$

which may be thought of as a homotopy theoretic model for the intersections of $P_{1}$ and $P_{2}$ in $N$. Indeed, a point in $E_{12}$ is a pair $\left(p_{1}, p_{2}\right)$ with $p_{i} \in P_{i}$ and a path between their images $f_{i}\left(p_{1}\right)$ in $N$, and we like to interpret this path as a plan for eliminating possible intersections of $f_{1}^{\prime}\left(p_{1}\right)$ with $f_{2}^{\prime}\left(p_{2}\right)$ for some link map $\left(f_{1}^{\prime}, f_{2}^{\prime}\right)$ homotopic to $\left(f_{1}, f_{2}\right)$ in $\operatorname{map}\left(P_{1}, N\right) \times \operatorname{map}\left(P_{2}, N\right)$. See Section 5.3 to see why the map $l_{2}$ deserves to be called the linking number. We will also show that a special case of this is the $\alpha$ invariant of Massey and Rolfsen [19], discussed in Section 5.3.

Theorem 1.3 There is a map

$l_{3}: \operatorname{hofiber}\left(\operatorname{Link}\left(P_{1}, P_{2}, P_{3} ; N\right) \rightarrow \mathcal{T}_{2} \operatorname{Link}\left(P_{1}, P_{2}, P_{3} ; N\right)\right) \rightarrow \Omega C_{3}\left(P_{1}, P_{2}, P_{3} ; N\right)$ which measures when the generalized Whitney trick fails.

The space $C_{3}\left(P_{1}, P_{2}, P_{3} ; N\right)$ is also a representing space for the cobordism of a space twisted by a bundle (described in Section 6). That space is the homotopy limit of the following diagram, which we call $E_{123}$.

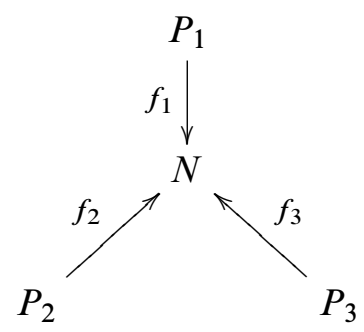

This too is a homotopy theoretic model for the intersections of the $P_{i}$ in $N$. Its importance will become apparent in Section 6 when we discuss how $\mathcal{T}_{2} \operatorname{Link}\left(P_{1}, P_{2}, P_{3} ; N\right)$ encodes the setup for the generalized Whitney trick: it tells one how to build generalized "Whitney disks" and the map $l_{3}$ measures intersections of the Whitney disks and intersections of $P_{k}$ with a Whitney disk meant to eliminate intersections of $P_{i}$ with $P_{j}$ for $i, j, k$ distinct.

One can ask whether or not these cobordism spaces describe these homotopy fibers in the sense that there is a highly connected map between them. This is indeed the case for $l_{2}$. The author and Tom Goodwillie [6] have shown:

Theorem 1.4 The map $l_{2}$ is $\left(2\left(n-p_{1}-p_{2}\right)-3\right)$-connected. 
The work of Scott [24] and Massey and Rolfsen [19] relates to this theorem, although it goes beyond the scope of this paper to discuss these results in any depth. The connectivity of $l_{3}$ is unknown at this time, and it may be a very difficult problem. We should note that the connectivity estimates for spaces of embeddings, due to Goodwillie and Klein [3] are extremely difficult to obtain, and it is possible this will be the case for link maps as well. We will discuss some of the difficulties in Section 4.

The two applications given for Theorem 1.2 and Theorem 1.3 are to identify the map of spaces given in Theorem 1.2 with the classical linking number, and to use Theorem 1.3 to prove that the Borromean rings are linked. Theorem 1.3 requires the use of manifolds with corners, and Section 2 addresses the relevant background. Section 3 gives the necessary background for cobordism spaces. Section 4 gives the necessary background for manifold calculus and contains the models for $\mathcal{T}_{1}$ Link and $\mathcal{T}_{2}$ Link which we utilize, as well as a brief discussion of the problem of analyticity of the functor Link $(-; N)$ and its importance. Section 5 is devoted to constructing the map of spaces appearing in Theorem 1.2, and Section 5.3 discusses why this is the same as the linking number in all of the classical cases. Section 6 is spent constructing the map of spaces in Theorem 1.3, and we use this map in Section 6.6 to prove that the Borromean rings are linked.

\subsection{Conventions}

We write $Q X$ for $\Omega^{\infty} \Sigma^{\infty} X$ where $\mathrm{X}$ is a based space, and $Q_{+} X$ for $Q\left(X_{+}\right)$when $X$ is unbased and where + denotes a disjoint basepoint. When we say a map is an equivalence, we mean it is a weak equivalence, unless otherwise noted. For a vector bundle $\xi$ over a space $\mathrm{X}$, we let $T(X ; \xi)$ denote the Thom space. We will use the same capital $T$ for tangent bundles, but this should cause no confusion. If $X$ is a finite dimensional unbased space equipped with vector bundles $\xi$ and $\eta$, choose a vector bundle monomorphism $\eta \rightarrow \epsilon^{i}$, and let $Q_{+}(X ; \xi-\eta)$ be $\Omega^{i} Q T\left(X ; \xi \oplus \epsilon^{i} / \eta\right)$. We write Spaces for the category of fibrant simplicial sets, and Top for the category of compactly generated topological spaces. Our mapping spaces are simplicial sets. For instance, $\operatorname{map}(M, N)$ is the simplicial set whose $k$-simplices are the fiber-preserving smooth maps of $M \times \Delta^{k} \rightarrow N \times \Delta^{k}$. By fiber-preserving we mean that $f_{k}(m, s)=(n, s)$. Other mapping spaces are translated to the category of simplicial sets in a similar manner.

\section{Manifolds with corners}

A manifold with corners is a generalization of the notion of a manifold with boundary. We will need to deal with the issue of giving a smooth structure to a manifold formed by 
gluing together several manifolds with corners to construct the map $l_{3}$ of Theorem 1.3, which will be discussed in Section 6. We have two goals. The first is to explain how to make a smooth manifold by gluing together smooth manifolds with corners, at least in the case where the codimension of the corners is small. The second is to explain how to glue together maps of vector bundles, given over a collection of manifolds with corners which glue together to form a smooth manifold, to make a vector bundle map over their union. The former will be used to show that the manifold defined in Section 6 is smooth, and the latter to show that vector bundle isomorphisms, given by transversality, can be glued together to form a vector bundle isomorphism over that manifold. Our definition of a manifold with corners is a modification of the definition of a smooth manifold given by Milnor in [21]. Following this work, we begin by discussing smooth functions on half-spaces.

Let $\mathbf{R}_{+}^{m}=[0, \infty)^{m}$. We can also think of $\mathbf{R}_{+}^{m}$ as a subset of $\mathbf{R}^{n}$ for $n>m$ by the inclusion of $\mathbf{R}^{m}$ in $\mathbf{R}^{n}$ as the first $m$ coordinates. For $0 \leq k \leq m$, let $\partial_{k} \mathbf{R}_{+}^{m}$ denote the subspace where at least $k$ of the coordinates are zero. We have $\partial_{m} \mathbf{R}_{+}^{m} \subset \cdots \subset$ $\partial_{k} \mathbf{R}_{+}^{m} \subset \cdots \subset \partial_{0} \mathbf{R}_{+}^{m}=\mathbf{R}_{+}^{m}$. We call $\partial_{k} \mathbf{R}_{+}^{m}$ the $k$-stratum of $\mathbf{R}_{+}^{m}$.

Definition 2.1 We say that a map $f: \mathbf{R}_{+}^{m} \rightarrow \mathbf{R}_{+}^{n}$ is smooth if it is the restriction of some smooth function $f^{\prime}: \mathbf{R}^{m} \rightarrow \mathbf{R}^{n}$.

Definition 2.2 A subset $M \subset \mathbf{R}^{k}$ is a smooth $m$-manifold with corners if each $x \in M$ has a neighborhood $U$ in $M$ that is diffeomorphic to an open subset $V$ in $\mathbf{R}_{+}^{m}$. The $k$-stratum (or codimension $k$ boundary) of $M$ is the set of all points in $M$ that correspond to points of $\partial_{k} \mathbf{R}_{+}^{m}$ under such a diffeomorphism, and we denote this set by $\partial_{k} M$

Let $M$ is a smooth $m$-manifold with corners, and let $k \leq m$ be fixed. If $M$ requires only charts of the form $\mathbf{R}_{+}^{i} \times \mathbf{R}^{m-i}$ for $i \leq k$, then we say $M$ has at most $k$-strata. The manifolds we will ultimately be interested in have at most 2 -strata.

\subsection{Tangent space}

To define the tangent space of a smooth manifold $M^{m} \subset \mathbf{R}^{k}$ at $x \in M$ we first pick a parametrization $g: U \rightarrow M$ of a neighborhood $g(U)$ of $x \in M$ with $g(u)=x$, and since $M \subset \mathbf{R}^{k}$ we may think of this as a map $g: \mathbf{R}^{m} \rightarrow \mathbf{R}^{k}$. We then define the tangent space $T_{x} M$ to be the image of $d g_{u}$. Now if $M$ is a manifold with corners whose interior is a smooth manifold, we can define as above the tangent space to $M$ at any point. In this case the parametrization $g$ is a smooth map $g: \mathbf{R}_{+}^{m} \rightarrow \mathbf{R}^{k}$, and by definition $g$ is the restriction of a smooth map $\mathbf{R}^{m} \rightarrow \mathbf{R}^{k}$. 


\subsection{Gluing and smooth structures}

We will only discuss gluing together manifolds with at most 2-strata, as that is all we require. The case $k=1$ is simple and well understood, so we review the relevant details here. A manifold with corners with only 1-strata is a manifold with boundary. Suppose one has two manifolds $M_{1}$ and $M_{2}$ with boundary and a diffeomorphism $j: \partial M_{1} \rightarrow \partial M_{2}$. Choose collars $\partial M_{1} \times[0,1) \subset M_{1}$ and $\partial M_{2} \times[0,1) \subset M_{2}$. We think of these as embeddings of $\partial M_{i} \times[0,1)$ in $M_{i}$ such that $(x, 0)$ maps to $x$. Then the union $M=M_{1} \cup_{j} M_{2}$ has the structure of a smooth manifold, since now every point in $M$ has a neighborhood diffeomorphic to $\mathbf{R}^{m}$. In particular, it is then clear what we mean by its tangent bundle $T M$. If we denote by $\partial$ the subset of $M$ corresponding to $j\left(\partial M_{1}\right)=\partial M_{2}$, then what we have shown is that $\partial$ has a neighborhood in $M$ homeomorphic to $\partial \times B^{1}$, where $B^{1}$ is an open 1 -disk.

One can generalize the existence of collars to manifolds with corners as follows.

Proposition 1 Let $M^{m}$ be a smooth manifold with corners with $k$-stratum $\partial_{k} M$. Then there exists a neighborhood $N$ of $\partial_{k} M$ in $M$ and a diffeomorphism $N \cong$ $\partial_{k} M \times[0,1)^{k}$.

One can mimic the proof using transversality Hirsch gives of the collaring theorem in [11]. We are going to use this to give a smooth structure to a closed topological manifold which is made from the union of smooth manifolds with corners. As we mentioned above, we will only discuss manifolds with at most $2-$ strata.

Proposition 2 Let $M_{1}, \ldots, M_{n}$ be manifolds with corners with at most 2-strata such that $\partial_{1} M_{i}=\partial_{1}^{i+1} M_{i} \cup \partial_{1}^{i-1} M_{i}$ and $\partial_{1}^{i+1} M_{i} \cap \partial_{1}^{i-1} M_{i}=\partial_{2} M_{i}$ for all $i$ (the subscripts are to be read modulo $n$ ). Let $j_{i, i+1} \partial_{1}^{i+1} M_{i} \rightarrow \partial_{1}^{i} M_{i+1}$ be diffeomorphisms, where again the subscripting integers are read modulo $n$. Suppose that the composition $j_{n, 1} \circ \cdots \circ j_{1,2}=$ id when restricted to $\partial_{2} M_{i}$. Then the union $M=\bigcup_{i} M_{i}$ can be given the structure of a smooth closed manifold.

The idea is to show that the image of the 2 -stratum $\partial_{2}$ in $M$ should have a neighborhood homeomorphic to $\partial_{2} \times B^{2}$, where $B^{2}$ is the open 2-disk.

Proof Divide $\mathbf{R}^{2}$ up into $n$ equal sectors $S_{i}$ for $i=1, \ldots n$. By a sector we mean the area between two rays meeting at the origin in angle $2 \pi / n$. Denote these rays by $\partial_{i-1} S_{i}$ and $\partial_{i+1} S_{i}$, for $i=1$ to $n$ modulo $n$.

Then $\partial_{2} M_{i}$ has a neighborhood $U_{i}$ in $M_{i}$ diffeomorphic to $\partial_{2} M_{i} \times[0,1)^{2}$ by Proposition 1 . We then choose diffeomorphisms $\partial_{2} M_{i} \times[0,1)^{2} \cong \partial_{2} M_{i} \times S_{i}$ in compatible 
with the diffeomorphisms $j_{i, i+1}$. By compatible we mean that the following diagram should commute.

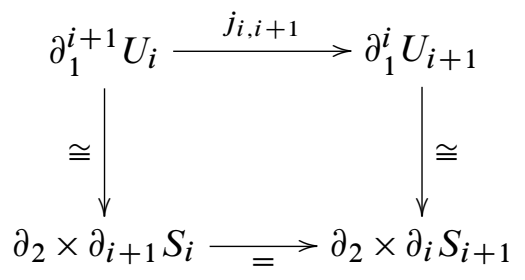

It follows that there is a neighborhood of $\partial_{2}$ in $M$ homeomorphic to $\partial_{2} \times B^{2}$. We use this homeomorphism to give $M$ its smooth structure. This gives $M$ a tangent bundle. Moreover, at each point in $\partial_{2}$, the restriction of the tangent bundle to each sector $S_{i}$ is the tangent bundle already given to $\partial_{2}$ on that sector by the inclusion of $M_{i}$.

\subsection{Vector bundles}

Proposition 3 Let $\xi$ and $\eta$ be vector bundles over a space $X$. Suppose we have manifolds $M_{1}$ and $M_{2}$, continuous proper maps $f_{i}: M_{i} \rightarrow X$ and isomorphisms $\phi_{i}: T M_{i} \oplus f_{i}^{*}(\xi) \rightarrow f_{i}^{*}(\eta)$ for $i=1,2$, and a diffeomorphism $j: \partial M_{1} \rightarrow \partial M_{2}$ satisfying $f_{1}=f_{2} \circ j$ on the boundary. Suppose that the restriction of $\phi_{1}$ and $j^{*} \phi_{2}$ to $\partial M_{1}$ and $\partial M_{2}$ respectively are homotopic. Then $M=M_{1} \cup_{j} M_{2}$ is a smooth manifold and there is an isomorphism $\phi: T M \oplus f^{*}(\xi) \rightarrow f^{*}(\eta)$ compatible with $\phi_{i}$ for $i=1,2$.

Proof Using collars of $\partial M_{i}$ and the diffeomorphism $j$ we can make a smooth manifold $M=M_{1} \cup_{j} M_{2}$ with a continuous map $f: M \rightarrow X$, as discussed in Section 2.2. The only thing left is to make a commutative diagram

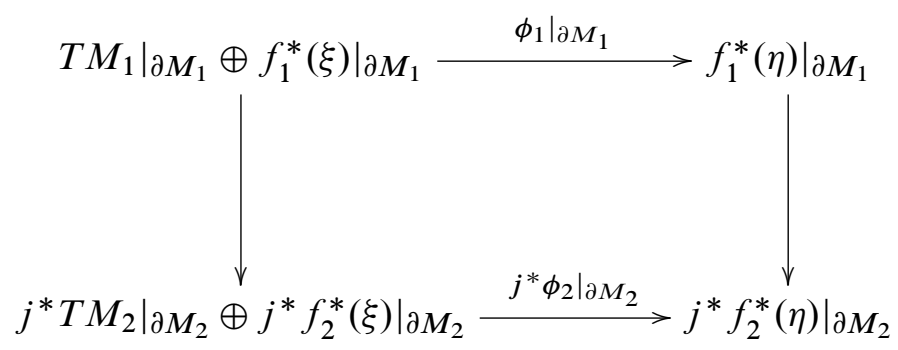

where the horizontal isomorphisms are the $\phi_{i}$, the right vertical isomorphism is the identity, and the leftmost isomorphism is given as follows. We write $\left.T M_{i}\right|_{\partial M_{i}}=$ $T \partial M_{i} \oplus \epsilon$ by identifying $\epsilon$ with $\nu\left(\partial M_{i} \subset M_{i}\right)$ and letting 1 correspond to the outer unit normal via some Riemannian metric on $M_{1}$, and let 1 correspond to the inner unit normal on $M_{2}$. Then the isomorphism between $T \partial M_{1} \oplus \epsilon$ and $j^{*} T \partial M_{2} \oplus j^{*} \epsilon$ is the 
obvious one (it is 1 on the $\epsilon$ part). If this diagram commutes, then the isomorphisms $\phi_{i}$ agree on $\partial M_{i}$, so we have produced a definite isomorphism $\phi: T M \oplus f^{*}(\xi) \rightarrow f^{*}(\eta)$ at all points $x \in M$. In fact, it is enough if the diagram above commutes up to homotopy. Let $\phi_{t}$ be the homotopy from $\phi_{1}$ to $\phi_{2}$. For notational convenience, we suppress the diffeomorphism $j$, and denote $\partial M_{i}$ by $\partial$. We have $M=\operatorname{colim}\left(M_{1} \leftarrow \partial \rightarrow M_{2}\right)$, and we have bundle isomorphisms on $M_{i}$ and a homotopy between them on $\partial$. If we set $M^{\prime}=\operatorname{hocolim}\left(M_{1} \leftarrow \partial \rightarrow M_{2}\right)$, then we have over each point in $M^{\prime}$ a definite isomorphism $T M \oplus f^{*}(\xi) \cong f^{*}(\eta)$. It is $\phi_{1}$ on $M_{1}, \phi_{2}$ on $M_{2}$, and $\phi_{t}$ on $\partial \times\{t\}$. The canonical map $M^{\prime} \rightarrow M$ is a homotopy equivalence, and we can pull back the bundle isomorphism on $M^{\prime}$ to $M$ by a homotopy inverse to get the desired bundle isomorphism.

Remark In our case the bundle isomorphisms are going to be given by transversality. That is, they will be induced by the derivatives of certain functions which define manifolds with corners, and we will need to check that they are homotopic.

As the proof of Proposition 3 indicates, the issues of giving a smooth structure to the union $M_{1} \cup M_{2}$ and that of gluing together the bundle isomorphisms can be dealt with separately. We next consider the process of forming a map of vector bundles over a space which is the union of other spaces by gluing together vector bundle maps given on the smaller pieces.

Our immediate goal is to describe a generalization of Proposition 3. This is the content of the next two propositions. The first describes the conditions under which one can form a vector bundle over a colimit of spaces given a vector bundle over each individual space. The second describes the conditions under which we can make a section of this bundle by gluing sections given in each space in the colimit.

Definition 2.3 Let $\alpha$ and $\beta$ be vector bundles over a space $Z$. Define $L_{\max }(\alpha, \beta)$ to be the space of vector bundle maps from $\alpha$ to $\beta$ over $Z$ such that the linear map of fibers is of maximal rank.

Let $\mathcal{C}$ be a small category, and suppose $\mathcal{X}: \mathcal{C} \rightarrow$ Top, is a covariant functor whose values we will denote $X(c)$, and assume that $X(c)$ is compact. Let $X(\mathcal{C})$ denote the diagram of spaces obtained from $\mathcal{X}$. Let $f_{c}: X(c) \rightarrow Y$ be maps for each $c \in \mathcal{C}$ such that the diagram $X(\mathcal{C}) \rightarrow Y$ commutes. Then $X=\operatorname{hocolim}_{\mathcal{C}} \mathcal{X}$ is a space with a map $f: X \rightarrow Y$ given by the $f_{c}$. The model for the homotopy colimit of a functor $F: \mathcal{C} \rightarrow$ Top, we have in mind for the homotopy colimit is the coequalizer

$$
\operatorname{hocolim}_{\mathcal{C}} F=\operatorname{coeq}\left(\coprod_{c \rightarrow c^{\prime}}\left|c^{\prime} \downarrow \mathcal{C}\right| \times F(c) \Longrightarrow \bigsqcup_{c}|c \downarrow \mathcal{C}| \times F(c)\right) .
$$


Proposition 4 Let $\mathcal{C}, \mathcal{X}, Y, \xi, \eta$, and $\left\{f_{c}\right\}_{c \in \mathcal{C}}$ be as above, and suppose $\xi$ and $\eta$ are vector bundles over $Y$. Consider the functor $\mathcal{E}: \mathcal{C} \rightarrow$ Top for which $\mathcal{E}(c)=$ $L_{\max }\left(f_{c}^{*} \xi, f_{c}^{*} \eta\right)$ as a bundle over $X(c)$ and the maps $\mathcal{E}(c) \rightarrow \mathcal{E}\left(c^{\prime}\right)$ are given by pullback of the map $X(c) \rightarrow X\left(c^{\prime}\right)$. Then $E=\operatorname{hocolim}_{\mathcal{C}} \mathcal{E}$ is a vector bundle over $X=\operatorname{hocolim}_{\mathcal{C}} \mathcal{X}$.

Proof This follows from the fact that the maps $\mathcal{E}(c) \rightarrow \mathcal{E}\left(c^{\prime}\right)$ are given by the pullback by $X(c) \rightarrow X\left(c^{\prime}\right)$ and the coequalizer definition of the homotopy colimit.

We wish to describe how to build a section of $E \rightarrow X$ from sections of $\mathcal{E}(c) \rightarrow \mathcal{X}(c)$. Let $\chi_{c \rightarrow c^{\prime}}: X(c) \rightarrow X\left(c^{\prime}\right)$ and $\Xi_{c \rightarrow c^{\prime}}: L_{\max }\left(f_{c}^{*} \xi, f_{c}^{*} \eta\right) \rightarrow L_{\max }\left(f_{c^{\prime}}^{*} \xi, f_{c^{\prime}}^{*} \eta\right)$ denote the maps given by the functors $\mathcal{X}$ and $\mathcal{E}$ respectively. Let $u_{c \rightarrow c^{\prime}}:\left|c^{\prime} \downarrow \mathcal{C}\right| \rightarrow|c \downarrow \mathcal{C}|$ be the induced map of realizations of under categories.

Proposition 5 Suppose we are given maps $\Phi_{c \rightarrow c^{\prime}}:\left|c^{\prime} \downarrow \mathcal{C}\right| \times X(c) \rightarrow L_{\max }\left(f_{c}^{*} \xi, f_{c}^{*} \eta\right)$. If these maps satisfy $\Phi\left(s^{\prime}, x\right)=\Phi\left(u\left(s^{\prime}\right), x\right)$ and $\Xi(\Phi(s, x)=\phi(s, \chi(x))$, then they piece together to form a section of $E \rightarrow X$.

Proof This follows immediately from the coequalizer definitions of $E$ and $X$.

We will be interested in the case when then $X(c)$ are manifolds with corners, $X:=$ $\operatorname{colim}_{\mathcal{C}} X$ has the structure of a smooth manifold, $X^{\prime}:=\operatorname{hocolim}_{\mathcal{C}} X$ and the map $X^{\prime} \rightarrow X$ is a homotopy equivalence so that we can pull back the bundle map obtained over $X^{\prime}$ by a homotopy inverse to $X$. In our case, the maps $\Phi_{c \rightarrow c^{\prime}}$ will be given by various homotopies, much as in Proposition 3. See Lemma 6.8.

\section{Cobordism spaces}

We begin with a very brief description of cobordism spaces which we will employ in our description of $\mathcal{T}_{i} \operatorname{Link}\left(P_{1}, \ldots, P_{k} ; N\right)$ for $i=1,2$. These spaces were used extensively in [22]. Identifying a good model for these spaces is necessary to define the maps in Section 5 and Section 6.

Let $X$ be a space, and $\xi$ and $\eta$ vector bundles on $X$. An element of the cobordism group $\Omega_{k}^{\xi-\eta}(X)$ is represented by a triple $\left(W^{k}, f, \phi\right)$ (sometimes denoted by just $W$ ) where $W$ is a $k$-dimensional smooth manifold embedded in $\mathbf{R}^{\infty}, f: W \rightarrow X$ is continuous and proper, and $\phi$ is a stable isomorphism $T W \oplus f^{*} \xi \cong f^{*} \eta$. The equivalence relation for representatives is the usual one defined by $(k+1)$-dimensional manifolds with boundary. We seek a space whose homotopy groups are the cobordism groups described above, and we call such a space a cobordism space. 
Definition 3.1 (Simplicial model for a cobordism space) The simplicial set $C_{\bullet}^{\xi-\eta}(X)$ has as its $k$-simplices the set $C_{k}=\left\{\left(W^{d+k}, f, \phi\right)\right\}$ where $d=\operatorname{dim}(\eta)-\operatorname{dim}(\xi), W$ is a smooth $(k+d)$-dimensional manifold embedded in $\mathbf{R}^{\infty} \times \Delta^{k}, W$ is transverse to $\mathbf{R}^{\infty} \times \partial_{S} \Delta^{k}$ for all nonempty subsets $S \subset\{0,1, \ldots, k\}, f: W \rightarrow X$ is continuous and proper, and $\phi: T W \oplus f^{*}(\xi) \rightarrow f^{*}(\eta)$ is a stable isomorphism.

The manifolds $W^{d+k} \subset \Delta^{k} \times \mathbf{R}^{\infty}$ should the conditioned. To be conditioned means that if we denote by $W_{t}$ the part of $W$ that sits over $t \in \Delta^{k}$, then $W_{t}$ should be independent of $t$ in a neighborhood of $\cup_{i} \partial_{i} \Delta^{k}$ (ie, $W_{t}=W^{\prime}$ for a fixed $W^{\prime}$, and $t$ near $\left.\bigcup_{i} \partial_{i} \Delta^{k}\right)$.

The face and degeneracy maps are induced by those of $\Delta^{\bullet}$. The $i$-th face map $d_{i}: C_{k} \rightarrow C_{k-1}$ is just the intersection of $W^{d+k}$ with the $i$-th face of $\Delta^{k}$. The $i-$ th degeneracy map $s_{i}: C_{k} \rightarrow C_{k+1}$ takes $W$ to the fiber product $W^{\prime}$

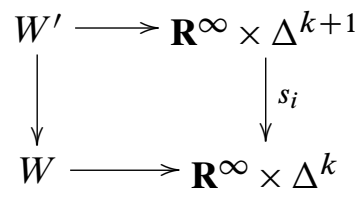

where $s_{i}$ is the $i$-th degeneracy for $\Delta^{\bullet}$. That $C_{\bullet}^{\xi-\eta}(X)$ satisfies the axioms for a simplicial set is straightforward; we are building on the usual simplicial structure on $\Delta^{k}$.

The next three propositions are established in [22].

Proposition $6 C_{\bullet}^{\xi-\eta}(X)$ is a Kan complex.

Proposition 7 There is an equivalence $C_{d+l}^{\xi-\eta}(X) \simeq \Omega^{l} C_{d}^{\xi-\eta}(X)$.

Remark This cobordism space is equivalent to $Q T(X ; \xi-\eta)$. To see the equivalence, consider the subcomplex of the total singular complex of $Q T(X ; \xi-\eta)$ consisting of those $k$-simplices $\kappa: \Delta^{k} \rightarrow \Omega^{n} \Sigma^{n}(T(\xi-\eta))$ that correspond to maps $\kappa^{\prime}: \Sigma^{n}\left(\Delta^{k}\right) \rightarrow$ $\Sigma^{n}(T(\xi-\eta))$ which are transverse to the zero section of $T(\xi-\eta)$. This subcomplex is equivalent to the full complex and the map $\kappa \mapsto \kappa^{\prime-1}(0)$ to the cobordism model is an equivalence. See Goodwillie [2] for a similar construction.

That $C$ • is a Kan complex ensures that the homotopy groups of its realization will be the cobordism groups we want. That is, $\pi_{k}\left|C_{d}^{\xi-\eta}(X)\right|=\Omega_{d+k}^{\xi-\eta}(X)$. The second proposition, together with the next, will be useful in explicitly identifying these cobordism groups in special cases, and will be used in our identification of the linking number in Section 5.3. 
Proposition $\mathbf{8}$ If $X$ is connected, the group $\Omega_{0}^{\xi-\eta}(X)$ is isomorphic with $\mathbf{Z}$ if $w(\xi)=$ $w(\eta)$, and $\mathbf{Z} / 2$ if $w(\xi) \neq w(\eta)$.

\section{Manifold calculus}

Manifold calculus, developed by Goodwillie and Weiss [7; 26] studies contravariant functors $F: \mathcal{O}(M) \rightarrow$ Spaces, where $\mathcal{O}(M)$ is the poset of open subsets of a smooth manifold $M$. Examples include $U \mapsto \operatorname{Emb}(U, N)$, the space of embeddings of $U$ in a smooth manifold $N, U \mapsto \operatorname{map}(U, X)$, the space of maps of $U$ to a space $X$, and $U \mapsto \operatorname{Imm}(U, N)$, the space of immersions of $U$ in a smooth manifold $N$. To such a functor $F$, the manifold calculus associates a tower of functors

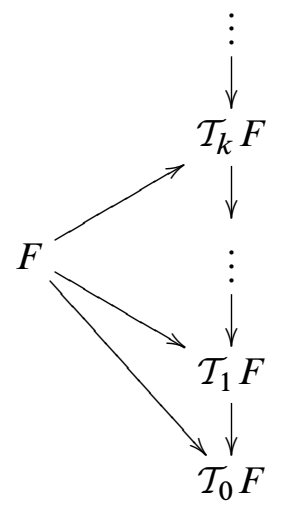

called the Taylor tower of $F$.

Definition 4.1 For a contravariant functor $F: \mathcal{O}(M) \rightarrow$ Spaces we define the $k-t h$ Taylor approximation to $F$, denoted $\mathcal{T}_{k} F: \mathcal{O}(M) \rightarrow$ Spaces, by

$$
\mathcal{T}_{k} F(U)=\operatorname{holim}_{V \in \mathcal{O}_{k}(U)} F(V) .
$$

Here $\mathcal{O}_{k}(U)$ is the subcategory of $\mathcal{O}(U)$ consisting of those open sets $V \subset U$ which are diffeomorphic to at most $k$ disjoint open balls.

Definition 4.2 We say that $F$ is polynomial of degree $\leq k$ if given pairwise disjoint closed subsets $A_{0}, A_{1}, \ldots, A_{k}$ of $U \in \mathcal{O}(M)$, the map

$$
F(U) \rightarrow \operatorname{holim}_{\varnothing \neq S \subset\{0,1, \ldots, k\}} F\left(U_{S}\right),
$$

where $U_{S}=U-\bigcup_{i \in S} A_{i}$, is a weak equivalence. We say $F$ is homogeneous of degree $k$ if additionally $\mathcal{T}_{k-1} F(U)$ is contractible for all $U$. 
For instance, the functors $U \mapsto \operatorname{map}(U, X)$ and $U \mapsto \operatorname{Imm}(U, N)$ are polynomial of degree $\leq 1$, and the functor $U \mapsto \operatorname{map}\left(U^{k}, X\right)$ is a polynomial of degree $\leq k$. See Weiss [26] for proofs. The next two theorems state that the functors $\mathcal{T}_{k} F$ are polynomial and are essentially determined by their values on special open sets.

Theorem 4.3 [26, Theorem 6.1] The cofunctor $\mathcal{T}_{k} F$ is polynomial of degree $\leq k$. If $F$ is already polynomial of degree $\leq k$, then $F \rightarrow \mathcal{T}_{k} F$ is an equivalence.

Remark 1 For a smooth manifold $X$ and a vector bundle $\xi \rightarrow X$, let $\Gamma(\xi, X)$ denote the space of sections. $\Gamma(\xi,-)$ is a polynomial of degree $\leq 1$ from $\mathcal{O}(X) \rightarrow$ Spaces. Hence, $\Gamma(\xi, X) \simeq \operatorname{holim}_{U \in \mathcal{O}(X)} \Gamma(\xi, U)$. In order to produce a section defined on all of $X$ is therefore enough to produce an open cover $\mathcal{U}$ of $X$, an element of $\Gamma(\xi, U)$ for each $U \in \mathcal{U}$, and homotopies between these sections on their intersections. We will use this fact in Lemma 6.7.

Theorem 4.4 [26, Theorem 5.1] Suppose that $\gamma: F_{1} \rightarrow F_{2}$ is a morphism of good cofunctors, and that $F_{i}$ is polynomial of degree $k$ for $i=1$, 2. If $\gamma: F_{1}(V) \rightarrow F_{2}(V)$ is a homotopy equivalence for all $V \in \mathcal{O}_{k}(M)$, then it is a homotopy equivalence for all $V \in \mathcal{O}(M)$.

From its definition we see that the values of $\mathcal{T}_{k} F$ are completely determined by its values on $\mathcal{O}_{k}(M)$, so Theorem 4.4 is not too surprising.

Finally, we state the classification theorem for homogeneous functors.

Theorem 4.5 [26, Theorem 8.1] Let $F$ be a homogeneous cofunctor of degree $k$. Then there is an equivalence

$$
F(V) \rightarrow \Gamma^{c}\left(p ;\left(\begin{array}{l}
V \\
k
\end{array}\right)\right)
$$

where $V \in \mathcal{O}(M)$, and $\Gamma^{c}\left(p ;\left(\begin{array}{l}V \\ k\end{array}\right)\right)$ is the space of compactly supported sections of a fibration $p: E \rightarrow\left(\begin{array}{l}V \\ k\end{array}\right)$.

Here "compactly supported" means equal to some fixed section, given by a choice of basepoint in $F(M)$, in some neighborhood of the fat diagonal. In particular, the functors $\mathcal{L}_{k} F=$ hofiber $\left(\mathcal{T}_{k} F \rightarrow \mathcal{T}_{k-1} F\right)$ are homogeneous, and we will make use of the classification theorem in Section 6.6. 


\subsection{Mapping space models for $\mathcal{T}_{1}$ Link and $\mathcal{T}_{2}$ Link}

Let $P_{1}, \ldots P_{k}$ be smooth closed compact manifolds of dimension $p_{1}, \ldots p_{k}$. We wish to apply manifold calculus to study the $\operatorname{space} \operatorname{Link}\left(P_{1}, \ldots P_{k} ; N\right)$. The functor in question is $\operatorname{Link}(-; N): \mathcal{O}\left(\coprod_{i=1}^{k} P_{i}\right) \rightarrow$ Spaces. Note that there is an equivalence of categories $\mathcal{O}\left(\coprod_{i=1}^{k} P_{i}\right) \rightarrow \prod_{i=1}^{k} \mathcal{O}\left(P_{i}\right)$ given by $U \mapsto\left(U_{1}, \ldots, U_{k}\right)$, where $U_{i}=U \cap P_{i}$. We now give mapping space models for $\mathcal{T}_{1} \operatorname{Link}\left(P_{1}, \ldots, P_{k} ; N\right)$ and $\mathcal{T}_{2} \operatorname{Link}\left(P_{1}, \ldots, P_{k} ; N\right)$ which will be used in the proof of the main theorems.

Proposition $9 \mathcal{T}_{1} \operatorname{Link}\left(P_{1}, \ldots, P_{k} ; N\right) \simeq \prod_{i=1}^{k} \operatorname{map}\left(P_{i}, N\right)$.

Proof Both of the functors in question are polynomial of degree $\leq 1$, and their values clearly agree when one $P_{i}$ is an open ball and the others are empty. Now consider the following diagram:

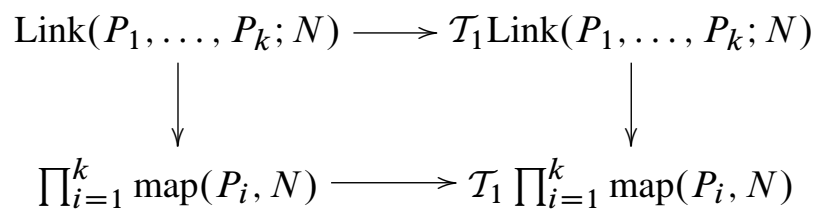

The lower horizontal arrow is an equivalence by Theorem 4.3, and the result now follows from Theorem 4.4.

Proposition $10 \mathcal{T}_{2} \operatorname{Link}\left(P_{1}, P_{2} ; N\right)$ is equivalent to the homotopy pullback of:

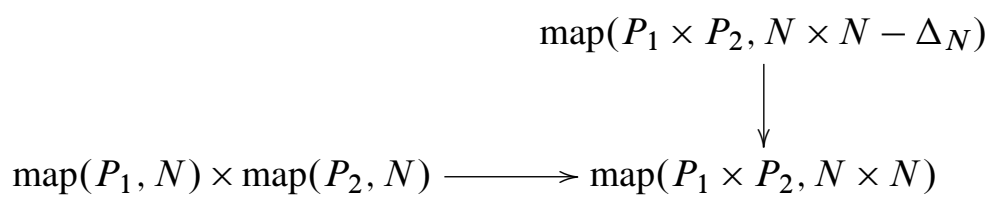

Proof Both are polynomials of degree less than or equal to 2, and we claim that the map from $\mathcal{T}_{2} \operatorname{Link}\left(P_{1}, P_{2} ; N\right)$ to the homotopy limit is an equivalence when $P_{1} \sqcup P_{2}$ is a manifold consisting of at most two open balls. The cases $P_{1}=\varnothing$ or $P_{2}=$ $\varnothing$ are straightforward to deal with. Without loss of generality, suppose $P_{2}=\varnothing$. Then, on the one hand, $P_{1}$ is a final object in $\mathcal{O}_{2}\left(P_{1}\right)$ and so $\mathcal{T}_{2} \operatorname{Link}\left(P_{1}, \varnothing ; N\right) \simeq$ $\operatorname{Link}\left(P_{1}, \varnothing ; N\right)=\operatorname{map}\left(P_{1}, N\right)$. On the other hand, the homotopy pullback diagram in question reduces to $\operatorname{map}\left(P_{1}, N\right)$ since $P_{1} \times \varnothing=\varnothing$.

If each of the $P_{i}$ is an open ball, then once again $P_{1} \sqcup P_{2}$ is a final object in $\mathcal{O}_{2}\left(P_{1} \sqcup P_{2}\right)$, and so $\mathcal{T}_{2} \operatorname{Link}\left(P_{1}, P_{2} ; N\right) \simeq \operatorname{Link}\left(P_{1}, P_{2} ; N\right) \simeq \operatorname{Link}(*, * ; N) \simeq N \times N-\Delta$. As for the homotopy pullback in question, clearly

$$
\operatorname{map}\left(P_{1} \times P_{2}, N \times N-\Delta_{N}\right) \simeq \operatorname{map}\left(* \times *, N \times N-\Delta_{N}\right)=N \times N-\Delta_{N},
$$


and so the diagram reduces to $\operatorname{holim}\left(N \times N \rightarrow N \times N \leftarrow N \times N-\Delta_{N}\right)$. The result follows from Theorem 4.4. Compare Theorem 1.3 of [5] in the case $k=2$.

Corollary 1 When $N=\mathbf{R}^{n}, \mathcal{T}_{2} \operatorname{Link}\left(P_{1}, P_{2} ; \mathbf{R}^{n}\right) \simeq \operatorname{map}\left(P_{1} \times P_{2}, S^{n-1}\right)$.

See Section 5.3 for applications of this to the classical linking number.

Corollary 2 The functor $\mathcal{T}_{2} \operatorname{Link}\left(P_{1}, \ldots, P_{k} ; N\right)$ is equivalent to product over $i<j$ of the homotopy pullbacks of:

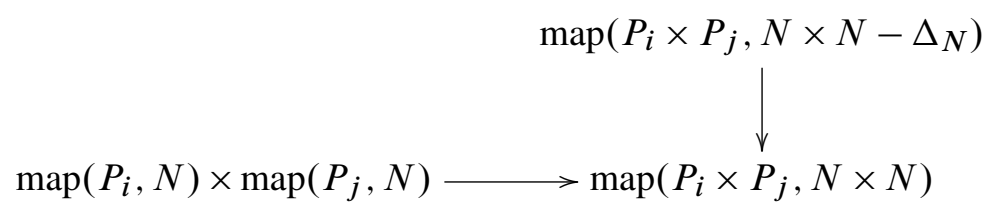

Proof This follows from Proposition 10 along with the fact that $\operatorname{map}(X \sqcup Y, Z)=$ $\operatorname{map}(X, Z) \times \operatorname{map}(Y, Z)$, by induction on $k$.

Hence, we may think of an element of $\mathcal{T}_{2} \operatorname{Link}\left(P_{1}, \ldots, P_{k} ; N\right)$ as a tuple $(\mathbf{f}, \mathbf{H}, \mathbf{F})$, where $\mathbf{f}=\left(\mathbf{f}_{\mathbf{1}}, \ldots, \mathbf{f}_{\mathbf{k}}\right)$ satisfies $f_{i} \in \operatorname{map}\left(P_{i}, N\right), \mathbf{F}=\left(\mathbf{F}_{\mathbf{1 2}}, \ldots, \mathbf{F}_{(\mathbf{k}-\mathbf{1}) \mathbf{k}}\right)$ satisfies $F_{i j} \in \operatorname{map}\left(P_{i} \times P_{j}, N \times N-\Delta_{N}\right)$, and $H_{i j}$ is a homotopy between $F_{i j}$ and $f_{i} \times f_{j}$.

\subsection{Analyticity}

One can ask whether these polynomial approximations actually approximate the functor, and whether or not $\operatorname{Link}\left(P_{1}, \ldots, P_{k} ; N\right) \rightarrow \operatorname{holim}_{j} \mathcal{T}_{j} \operatorname{Link}\left(P_{1} \ldots, P_{k} ; N\right)$ is an equivalence. If so, we would say that the polynomial approximations converge to the original functor and that the functor is analytic. For the functor $\operatorname{Link}(-; N)$, this appears to be a very difficult problem.

For smooth manifolds $M^{m}$ and $N^{n}$ and the functor $U \mapsto \operatorname{Emb}(U, N)$ for $U \subset M$, Goodwillie and Klein [3] prove that if $n-m-2>0$, then the maps $\operatorname{Emb}(M, N) \rightarrow$ $\mathcal{T}_{k} \operatorname{Emb}(M, N)$ have connectivity increasing with $k$, and so the Taylor tower converges to this functor, and $\operatorname{Emb}(-, N)$ is analytic with radius of convergence $n-m-2$. The proof of these connectivity estimates is very difficult, and involves, among other things, diffeomorphisms and their relationship (via the isotopy extension theorem) to embeddings. There is no analog for link maps of the isotopy extension theorem, because the restriction map $\operatorname{Link}\left(K_{2} ; N\right) \rightarrow \operatorname{Link}\left(K_{1} ; N\right)$ for compact sets $K_{1} \subset K_{2} \subset P_{1} \sqcup P_{2}$ is not in general a fibration. For example, let $P_{1}=\{p\}$ be a single point, $P_{2}=\left\{q_{1}, q_{2}\right\}$, and let $K_{1}=P_{2}$ and $K_{2}=P_{1} \sqcup P_{2}$. This makes it difficult to analyze homotopy fibers of such restrictions, unlike the case for embeddings, where the isotopy extension theorem says that the restriction map is a fibration. 


\section{Linking manifolds and quadratic obstructions}

In this section we are going to examine the difference between $\operatorname{Link}\left(P_{1}, P_{2} ; N\right)$ and $\mathcal{T}_{1} \operatorname{Link}\left(P_{1}, P_{2} ; N\right)$. It should be clear from what follows that for $\operatorname{Link}\left(P_{1}, \ldots, P_{k} ; N\right)$ we make $\left(\begin{array}{c}k \\ 2\end{array}\right)$ linking manifolds, one for each pair $(i, j)$ with $i<j$. Recall from Proposition 9 that $\mathcal{T}_{1} \operatorname{Link}\left(P_{1}, P_{2} ; N\right) \simeq \operatorname{map}\left(P_{1}, N\right) \times \operatorname{map}\left(P_{2}, N\right)$.

Let $\left(f_{1}, f_{2}\right) \in \operatorname{Link}\left(P_{1}, P_{2} ; N\right)$ be the basepoint, and let

$$
\alpha \in \operatorname{hofiber}\left(\operatorname{Link}\left(P_{1}, P_{2} ; N\right) \rightarrow \operatorname{map}\left(P_{1}, N\right) \times \operatorname{map}\left(P_{2}, N\right)\right) .
$$

This is a map $\alpha: I \rightarrow \operatorname{map}\left(P_{1}, N\right) \times \operatorname{map}\left(P_{2}, N\right)$ such that $\alpha(0)=\left(f_{1}, f_{2}\right)$, and $\alpha(1) \in \operatorname{Link}\left(P_{1}, P_{2} ; N\right)$. We will write $\alpha(t)=\left(f_{1, t}, f_{2, t}\right)$ so that $f_{1,0}=f_{1}$ and $f_{2,0}=f_{2}$.

\subsection{Quadratic cobordism space model}

The constructions in this section were inspired by a very similar construction in Section 1.4 of Goodwillie, Klein and Weiss [5]. Compare also Klein and Williams [12].

Definition 5.1 $E_{12}=\operatorname{holim}\left(P_{1} \stackrel{f_{1}}{\rightarrow} N \stackrel{f_{2}}{\leftarrow} P_{2}\right)$.

Hence $E_{12}$ is the set of all $\left(x_{1}, x_{2}, \omega\right)$ where $\left(x_{1}, x_{2}\right) \in P_{1} \times P_{2}, \omega:[-1,1] \rightarrow N$, $\omega(-1)=f_{1}\left(x_{1}\right)$ and $\omega(1)=f_{2}\left(x_{2}\right)$.

$E_{12}$ has maps to $P_{1} \times P_{2}$ and $N$ given by projection and evaluation of $\omega$ at 0 . Hence we may pull back $T P_{1} \times T P_{2}$ and $T N$ to $E_{12}$. From this data we can make a cobordism space $C_{\bullet}^{T N-T P_{1} \times T P_{2}}\left(E_{12}\right)$. For us, $d=p_{1}+p_{2}-n$, so that a $0-$ simplex in this space is a manifold of dimension $\left(p_{1}+p_{2}-n\right)$. We will abbreviate this space by $C_{2}\left(P_{1}, P_{2} ; N\right)$. It is equivalent to $Q T\left(E_{12} ; T N-T P_{1} \times T P_{2}\right)$ by the Pontryagin-Thom construction.

We are now going to produce a path in $C_{2}\left(P_{1}, P_{2} ; N\right)$ from our choice of $\alpha \in$ hofiber $\left(\operatorname{Link}\left(P_{1}, P_{2} ; N\right) \rightarrow \operatorname{map}\left(P_{1}, N\right) \times \operatorname{map}\left(P_{2}, N\right)\right)$. Recall from above the notation $\alpha_{t}=\left(f_{1, t}, f_{2, t}\right)$. We may regard $f_{1, t} \times f_{2, t}: P_{1} \times P_{2} \rightarrow N \times N$ as a map $F: P_{1} \times P_{2} \times I \rightarrow N \times N$. We may assume that $F$ is transverse to the diagonal $\Delta_{N} \subset N \times N$.

Definition 5.2 $L_{\alpha}=F^{-1}\left(\Delta_{N}\right)$.

Proposition $11 L$ is a smooth closed compact $\left(p_{1}+p_{2}+1-n\right)$-dimensional manifold with stable normal bundle $T N-T P_{1} \times T P_{2}$. Also, $L$ defines an element of $\Omega Q T\left(E_{12} ; T N-T P_{1} \times T P_{2}\right)$.

Proof This follows from transversality. 
$L_{\alpha} \subset E_{12} \times[0,1]$ is the subset of all $\left(x_{1}, x_{2}, \omega, t\right)$ such that $f_{1, t}\left(x_{1}\right)=f_{2, t}\left(x_{2}\right)$, where $\omega$ is the path

$$
\omega(s)= \begin{cases}f_{1,(1+s) t} & \text { if }-1 \leq s \leq 0 \\ f_{2,(1-s) t} & \text { if } 0 \leq s \leq 1 .\end{cases}
$$

Thus $L_{\alpha}$ determines a point in $\Omega C_{2}\left(P_{1}, P_{2} ; N\right)$ the space whose 0 -simplices are cobordisms of $\varnothing$ with itself. This works for arbitrary maps of a $k$-simplex into hofiber(Link $\left.\left(P_{1}, P_{2} ; N\right) \rightarrow \operatorname{map}\left(P_{1}, N\right) \times \operatorname{map}\left(P_{2}, N\right)\right)$, producing a $k$-simplex in $\Omega C_{2}\left(P_{1}, P_{2} ; N\right)$, or equivalently a $k$-simplex in $\Omega Q T\left(E_{12} ; T N-T P_{1} \times T P_{2}\right)$. We need to note that the subcomplex of maps which satisfy the necessary transversality condition to define $L_{\alpha}$ are homotopy equivalent to the full complex (see, for instance, Hypothesis 3.18 of [2]). This proves Theorem 1.2, restated here for convenience.

\section{Theorem 5.3 There is a map of spaces}

$$
l: \operatorname{hofiber}\left(\operatorname{Link}\left(P_{1}, P_{2} ; N\right) \rightarrow \operatorname{map}\left(P_{1}, N\right) \times \operatorname{map}\left(P_{2}, N\right)\right) \rightarrow \Omega C_{2}\left(P_{1}, P_{2} ; N\right)
$$

which sends $\alpha$ to $L_{\alpha}$, and can be described as the "generalized linking number".

It is not necessary to assume that the basepoint $\left(f_{1}, f_{2}\right)$ is in the image of the space of link maps. In this case the above would be modified only slightly. Instead of cobordisms of the empty set with itself, we would have nullcobordisms of the intersection of $f_{1}\left(P_{1}\right)$ with $f_{2}\left(P_{2}\right)$. As mentioned in the introduction, Theorem 1.4, to appear in [6], tells us the connectivity of this map.

\subsection{Properties of the linking manifold}

We have a map

$$
\operatorname{hofiber}\left(\operatorname{Link}\left(P_{1}, P_{2} ; N\right) \rightarrow \operatorname{map}\left(P_{1}, N\right) \times \operatorname{map}\left(P_{2}, N\right)\right) \rightarrow \Omega C_{2}\left(P_{1}, P_{2} ; N\right) \text {. }
$$

As above, for each $\alpha \in \operatorname{hofiber}\left(\operatorname{Link}\left(P_{1}, P_{2} ; N\right) \rightarrow \operatorname{map}\left(P_{1}, N\right) \times \operatorname{map}\left(P_{2}, N\right)\right)$, we write $\alpha_{t}=\left(f_{1, t}, f_{2, t}\right)$ and from this produce an element of $\Omega C_{2}\left(P_{1}, P_{2} ; N\right)$, which is represented by a smooth closed compact manifold $L_{\alpha}$ of dimension $\left(p_{1}+p_{2}-n+1\right)$, called the linking manifold of $\alpha$, and it plays the role of the linking number of $\left(f_{1}, f_{2}\right)$.

Clearly the linking manifold depends both on the choice of basepoint $\left(f_{1}, f_{2}\right)$ and the choice of $\alpha \in \operatorname{hofiber}\left(\operatorname{Link}\left(P_{1}, P_{2} ; N\right) \rightarrow \operatorname{map}\left(P_{1}, N\right) \times \operatorname{map}\left(P_{2}, N\right)\right)$. The dependence on the basepoint is unavoidable, but in many cases, the linking manifold does not depend on the choice of path $\alpha$, only its values at 0 and 1. See Section 5.2.1 for more. 
5.2.1 Dependence on element of the homotopy fiber Let us now examine the extent to which the linking manifold depends on the path $\alpha$. These results are simple but useful corollaries of Theorem 1.2.

Proposition 12 Let $\alpha, \beta \in \operatorname{hofiber}\left(\operatorname{Link}\left(P_{1}, P_{2} ; N\right) \rightarrow \operatorname{map}\left(P_{1}, N\right) \times \operatorname{map}\left(P_{2}, N\right)\right)$ and that $\alpha(1)=\beta(1)$. If $\alpha$ and $\beta$ are homotopic, then the linking manifolds $L_{\alpha}$ and $L_{\beta}$ are cobordant.

Corollary 3 If $\operatorname{map}\left(P_{1}, N\right)$ and $\operatorname{map}\left(P_{2}, N\right)$ are simply connected, then the cobordism class of the linking manifold of $\left(f_{1,1}, f_{2,1}\right)$ only depends on the choice of basepoint $\left(f_{1}, f_{2}\right)$.

Proof If $\operatorname{map}(X, Y)$ is simply connected, then any two paths in $\operatorname{map}(X, Y)$ sharing the same endpoints are homotopic through paths fixed at their endpoints. The result follows from Proposition 12.

Of course, $\operatorname{map}\left(X, \mathbf{R}^{k}\right)$ is contractible. In general, it is straightforward to show that $\operatorname{map}(X, Y)$ is $(\operatorname{conn}(Y)-\operatorname{dim}(X))$-connected by considering it as the space of sections of a trivial bundle. If we choose another basepoint $\left(f_{1}^{\prime}, f_{2}^{\prime}\right) \in \operatorname{Link}\left(P_{1}, P_{2} ; N\right)$ in the same path component as $\left(f_{1}, f_{2}\right)$, then the linking manifold remains unchanged. In particular, this is the case if $N$ is connected and $\left(f_{1}, f_{2}\right)$ and $\left(f_{1}^{\prime}, f_{2}^{\prime}\right)$ send $P_{1}$ and $P_{2}$ to a distinct pair of points in $N$.

Remark In the work of Chernov and Rudyak [1], the authors have a way to produce a linking number for a given link from a choice of the unlink in the case where $p_{1}+$ $p_{2}+1=n$. It is an element of a cobordism group modulo a group of indeterminacies. Clearly our construction depends also on a choice of path in the $\operatorname{space} \operatorname{map}\left(P_{1}, N\right) \times$ $\operatorname{map}\left(P_{2}, N\right)$. In the case where $\operatorname{map}\left(P_{1}, N\right) \times \operatorname{map}\left(P_{2}, N\right)$ is simply connected, this group of indeterminacies vanishes. This group of indeterminacies also vanishes in other special situations. See especially Section 6 of [1] for details.

\subsection{The classical linking number}

Suppose $f_{1}: S^{p_{1}} \rightarrow \mathbf{R}^{p_{1}+p_{2}+1}$ and $f_{2}: S^{p_{2}} \rightarrow \mathbf{R}^{p_{1}+p_{2}+1}$ are smooth maps with disjoint images. This produces a map

$$
f_{1} \times f_{2}: S^{p_{1}} \times S^{p_{2}} \rightarrow \mathbf{R}^{p_{1}+p_{2}+1} \times \mathbf{R}^{p_{1}+p_{2}+1}-\Delta_{\mathbf{R}^{p_{1}+p_{2}+1}},
$$

and by subtraction and retraction, a map $F: S^{p_{1}} \times S^{p_{2}} \rightarrow S^{p_{1}+p_{2}}$. One classical definition of the linking number of $f_{1}$ and $f_{2}$ is the degree of this map, which may 
be computed by computing the cardinality of the inverse image of a regular value $x \in S^{p_{1}+p_{2}}$ with signs. The sign convention is as follows. Give an orientation to $S^{p_{1}}$, $S^{p_{2}}$, and $S^{p_{1}+p_{2}+1}$. Each point $\left(x_{1}, x_{2}\right)$ in $F^{-1}(x) \subset S^{p_{1}} \times S^{p_{2}}$ is assigned the value +1 or -1 according to whether the derivative $D F: T_{x_{1}} S^{p_{1}} \times T_{x_{2}} S^{p_{2}} \rightarrow T_{x} S^{p_{1}+p_{2}}$ is orientation preserving or reversing respectively. We will examine this is a slightly more general context where the $p_{1}$-sphere and the $p_{2}$-sphere are replaced by smooth closed compact manifolds $P_{1}$ and $P_{2}$ of dimensions $p_{1}$ and $p_{2}$ respectively. If these manifolds are oriented, then the sign convention is the same as that above, and if either of them is not oriented, then the linking number is an integer mod 2.

Let us first deal with the right way to count the linking number from our perspective. By Proposition $8, \Omega_{0}^{T \mathbf{R}^{p_{1}+p_{2}+1}-T P_{1} \times T P_{2}}\left(E_{12}\right)$ is $\mathbf{Z}$ if $P_{1}$ and $P_{2}$ are oriented, and $\mathbf{Z} / 2$ otherwise.

Consider the map $l_{2}$ : hofiber $\left(\operatorname{Link}\left(P_{1}, P_{2} ; \mathbf{R}^{p_{1}+p_{2}+1}\right) \rightarrow \operatorname{map}\left(P_{1}, \mathbf{R}^{p-1+p_{1}+1}\right) \times\right.$ $\left.\operatorname{map}\left(P_{2}, \mathbf{R}^{p_{1}+p_{2}+1}\right)\right) \rightarrow \Omega C_{2}\left(P_{1}, P_{2} ; \mathbf{R}^{p_{1}+p_{2}+1}\right)$. Hence for each

$\alpha \in \operatorname{hofiber}\left(\operatorname{Link}\left(P_{1}, P_{2} ; \mathbf{R}^{p_{1}+p_{2}+1}\right) \rightarrow \operatorname{map}\left(P_{1}, \mathbf{R}^{p_{1}+p_{2}+1}\right) \times \operatorname{map}\left(P_{2}, \mathbf{R}^{p_{1}+p_{2}+1}\right)\right.$

we get a manifold $L$ of dimension 0 . Taking the induced map on $\pi_{0}$ and using Proposition 7, we see that $l(\alpha)$ determines a class $\left[L_{\alpha}\right]$ in $\Omega_{0}^{T N-T P_{1} \times T P_{2}}\left(E_{12}\right)$.

Next we will show that our definition agrees with the classical one when the basepoint is chosen so that the images of $f_{1}$ and $f_{2}$ lie inside disjoint open balls, and that the choice of lift $\alpha$ is immaterial.

Let

$\alpha, \beta \in \operatorname{hofiber}\left(\operatorname{Link}\left(P_{1}, P_{2} ; \mathbf{R}^{p_{1}+p_{2}+1}\right) \rightarrow \operatorname{map}\left(S^{p}, \mathbf{R}^{p+q+1}\right) \times \operatorname{map}\left(S^{q}, \mathbf{R}^{p+q+1}\right)\right)$ satisfy $\alpha(1)=\beta(1)$. We write $\alpha(t)=\left(f_{1, t}, f_{2, t}\right)$ as before, and $\beta(t)=\left(f_{1, t}^{\prime}, f_{2, t}^{\prime}\right)$. Let $H$ be the straight line homotopy between them. That is,

$$
H(s, t)=\left(s f_{1, t}+(1-s) f_{1, t}^{\prime}, s f_{2, t}+(1-s) f_{2, t}^{\prime}\right) .
$$

Then $H$ gives rise to a cobordism between $L_{\alpha}$ and $L_{\beta}$.

Consider the composite map

$P_{1} \times P_{2} \stackrel{f_{1} \times f_{2}}{\longrightarrow} \mathbf{R}^{p_{1}+p_{2}+1} \times \mathbf{R}^{p_{1}+p_{2}+1}-\Delta_{\mathbf{R}^{p_{1}+p_{2}+1}} \stackrel{d}{\rightarrow} \mathbf{R}^{p_{1}+p_{2}+1}-\{0\} \stackrel{r}{\rightarrow} S^{p_{1}+p_{2}}$.

As we mentioned, the linking number is the degree of the composed map. As a manifold, it is the inverse image of a regular value $x \in S^{p_{1}+p_{2}}$, counted with signs (equivalently, framed) as described above. By inspection, this is the solutions to the equation $f_{1}(x)-$ $t z=f_{2}(y)$, where $t>0$ and $z$ is a regular value of the composite above. By 
compactness we and reparametrization of the time coordinate we can assume all these solutions occur for $t \in[0,1]$. Now we can interpret $\left(f_{1}(x)-t z, f_{2}(y)\right)$ as an element of hofiber $\left(\operatorname{Link}\left(P_{1}, P_{2} ; \mathbf{R}^{p_{1}+p_{2}+1}\right) \rightarrow \operatorname{map}\left(S^{p}, \mathbf{R}^{p+q+1}\right) \times \operatorname{map}\left(S^{q}, \mathbf{R}^{p+q+1}\right)\right)$, and clearly $\left(f_{1}(x)-z, f_{2}(y)\right)$ is the standard unlink: the images of the two spheres lie in disjoint open balls.

This is clearly related to the $\alpha$ invariant of Massey and Rolfsen [19]. From maps $f_{1}: S^{p_{1}} \rightarrow \mathbf{R}^{p_{1}+p_{2}+1}$ and $f_{2}: S^{p_{2}} \rightarrow \mathbf{R}^{m}$ with disjoint images we can construct a map $F: S^{p_{1}} \times S^{p_{2}} \rightarrow S^{m-1}$ as above. If $p_{1}, p_{2} \leq m-2$, then the homotopy classes of maps $S^{p_{1}} \times S^{p_{2}} \rightarrow S^{m-1}$ are in bijective correspondence with elements of $\pi_{p_{1}+p_{2}}\left(S^{m-1}\right)$, and Massey and Rolfsen define $\alpha(F)$ to be the element of $\pi_{p_{1}+p_{2}}\left(S^{m-1}\right)$ corresponding to the homotopy class of $F$.

5.3.1 Linking in $S^{p_{1}+p_{2}+1}$ By imposing some mild dimensional assumptions we can argue by using our work in the previous section that the classical linking number for linking in spheres agrees with ours. The key observation is that made in the comments following Corollary 3: $\operatorname{map}(X, Y)$ is $(\operatorname{conn}(Y)-\operatorname{dim}(X))-$ connected. Since $S^{p_{1}+p_{2}+1}$ is $\left(p_{1}+p_{2}\right)$-connected, $\operatorname{map}\left(P_{1}, S^{p_{1}+p_{2}+1}\right)$ and $\operatorname{map}\left(P_{2}, S^{p_{1}+p_{2}+1}\right)$ are simply connected provided $p_{1}, p_{2} \geq 1$. In order to use the straight line homotopy as we did before, we need to make sure that every 1-simplex (homotopy of maps) in $\operatorname{map}\left(P_{1}, S^{p_{1}+p_{2}+1}\right)$ and $\operatorname{map}\left(P_{2}, S^{p_{1}+p_{2}+1}\right)$ misses a point (so that they may be considered as maps to $\mathbf{R}^{p_{1}+p_{2}+1}$ and thus we can use the straight line homotopy between them). This will happen provided $p_{1}+1<p_{1}+p_{2}+1$ and $p_{2}+1<p_{1}+p_{2}+1$, or $p_{1}, p_{2} \geq 1$.

\section{Cubic obstructions}

In this section we seek to describe the difference between $\operatorname{Link}\left(P_{1}, \ldots, P_{k} ; N\right)$ and $\mathcal{T}_{2} \operatorname{Link}\left(P_{1}, \ldots, P_{k} ; N\right)$ in a similar fashion to the way we described the difference between $\operatorname{Link}\left(P_{1}, \ldots, P_{k} ; N\right)$ and $\mathcal{T}_{1} \operatorname{Link}\left(P_{1}, \ldots, P_{k} ; N\right)$ in the previous section. That is, we will give a map from hofiber $\left(\operatorname{Link}\left(P_{1}, \ldots, P_{k} ; N\right) \rightarrow \mathcal{T}_{2} \operatorname{Link}\left(P_{1}, \ldots, P_{k} ; N\right)\right)$ to a cobordism space. As an example, we will use this map to show that the Borromean rings are linked in Section 6.6. We begin by giving a cobordism space model for hofiber( $\left.\operatorname{Link}\left(P_{1}, P_{2}, P_{3} ; N\right) \rightarrow \mathcal{T}_{2} \operatorname{Link}\left(P_{1}, P_{2}, P_{3} ; N\right)\right)$, and then go about constructing a manifold in a similar manner to that above.

\subsection{Cubic cobordism space model}

Choose a basepoint $(\mathbf{f}, \mathbf{F}, \mathbf{H}) \in \mathcal{T}_{2} \operatorname{Link}\left(P_{1}, P_{2}, P_{3} ; N\right)$. As before $\mathbf{f}=\left(f_{1}, f_{2}, f_{3}\right)$, $\mathbf{F}=\left(F_{12}, F_{23}, F_{31}\right)$, and $\mathbf{H}=\left(H_{12}, H_{23}, H_{31}\right) . H_{i j}: P_{i} \times P_{j} \times I \rightarrow N \times N$ is a homotopy between $H_{i j}(0)=\left(f_{i}, f_{j}\right)$ and $H_{i j}(1)=F_{i j}$. 
Consider the following diagram $\mathcal{D}$ :

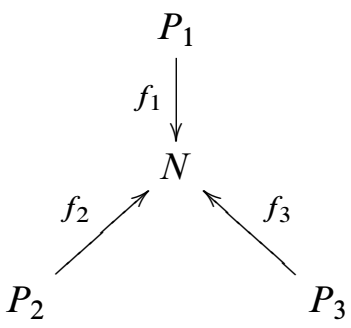

Definition 6.1 Define $E_{123}=\operatorname{holim}(\mathcal{D})$.

Thus a point in $E_{123}$ is a tuple $\left(x_{1}, x_{2}, x_{3}, \omega_{1}, \omega_{2}, \omega_{3}\right)$, where $x_{i} \in P_{i}$, and $\omega_{i}: I \rightarrow N$ is a path such that $\omega_{i}(0)=f_{i}\left(x_{i}\right)$, and $\omega_{1}(1)=\omega_{2}(1)=\omega_{3}(1)$ for $i=1,2,3$. Evidently $E_{123}$ has a map to $P_{1} \times P_{2} \times P_{3}$ given by projection, which we use to pull back $T P_{1} \times T P_{2} \times T P_{3}$ to $E_{123}$. We also have a map $E_{123} \rightarrow N \times N$, defined as follows. There is a map

$$
E_{123} \rightarrow \prod_{i \neq j} \operatorname{holim}\left(P_{i} \stackrel{f_{i}}{\rightarrow} N \stackrel{f_{j}}{\leftarrow} P_{j}\right)
$$

given by $\left(\omega_{1}, \omega_{2}, \omega_{3}\right) \mapsto\left(\omega_{1} \cdot \omega_{2}^{-1}, \omega_{2} \cdot \omega_{3}^{-1}, \omega_{3} \cdot \omega_{1}^{-1}\right)$, where $\cdot$ indicates path multiplication. If we reparametrize the resulting paths to be defined on $[-1,1]$ then the map to $E_{123} \rightarrow N \times N$ can be defined as the composition of this map with the evaluation $\left(\omega_{1} \cdot \omega_{2}^{-1}, \omega_{2} \cdot \omega_{3}^{-1}, \omega_{3} \cdot \omega_{1}^{-1}\right) \mapsto\left(\omega_{1} \cdot \omega_{2}^{-1}(0), \omega_{2} \cdot \omega_{3}^{-1}(0)\right)$, and we use this map to pull back $T N \times T N$ to $E_{123}$.

Definition 6.2 Define $C_{3}\left(P_{1}, P_{2}, P_{3} ; N\right)=C_{\bullet}^{T N \times T N-T P_{1} \times T P_{2} \times T P_{3}}\left(E_{123}\right)$.

In this case, $d=p_{1}+p_{2}+p_{3}-2 n$, but for indexing purposes, it is more convenient to shift this down by 2 (this could be done honestly by subtracting twice the trivial 1 -dimensional bundle, but that is rather cumbersome notationally). Thus, a 0 -simplex in this space will be represented by a manifold of dimension $p_{1}+p_{2}+p_{3}-2 n+2$. Note that $C_{3}$ is equivalent to $Q T\left(E_{123} ; T N \times T N-T P_{1} \times T P_{2} \times T P_{3}\right)$ by the Pontryagin-Thom construction.

\subsection{Whitney circles and Whitney disks}

Proposition 10 tells us that we may think of an element of $\mathcal{T}_{2} \operatorname{Link}\left(P_{1}, P_{2} ; N\right)$ as a triple $\left(\left(f_{1}, f_{2}\right), F_{12}, H_{12}\right)$ where $f_{i}: P_{i} \rightarrow N, F_{12}: P_{1} \times P_{2} \rightarrow N \times N-\Delta_{N}$, and $H_{12}: P_{1} \times P_{2} \times I \rightarrow N \times N$ is a homotopy from $f_{1}$ to $f_{2}$. Suppose $H_{12}$ is transverse 
to $\Delta_{N}$. Then $D_{12}=H_{12}^{-1}\left(\Delta_{N}\right)$ is a $\left(p_{1}+p_{2}+1-n\right)$-dimensional submanifold of $P_{1} \times P_{2} \times I$ which we call the Whitney circle. The reason for this terminology is that in case $D_{12}$ is 1 -dimensional, the images under $f_{i}$ of the projections of $D_{12}$ to $P_{i}$ for $i=1,2$ form a bent circle in $N$. This circle bounds a disk in $N$ as follows. For each $\left(x_{1}, x_{2}, t_{0}\right) \in D_{12}$ we give a path in $N$ from $f_{1}\left(x_{1}\right)$ to $f_{2}\left(x_{2}\right)$ by going from $f_{1}\left(x_{1}\right)=p_{1} H_{12}\left(x_{1}, x_{2}, 0\right)$ to $p_{1} H_{12}\left(x_{1}, x_{2}, t_{0}\right)=p_{2} H_{12}\left(x_{1}, x_{2}, t_{0}\right)$ by letting $t$ run from 0 to $t_{0}$, and then to $f_{2}\left(x_{2}\right)$ by letting $t$ run from $t_{0}$ back down to 0 . We call this disk (and its higher-dimensional counterpart) the Whitney disk. Our obstruction manifold measures the intersections of the Whitney circles, and the intersections of the manifolds themselves with the Whitney disks. Compare an identical description in Schneiderman and Teichner [23] in the case of 2-spheres in a 4-manifold.

\subsection{Construction of the obstruction manifold}

We will construct the obstruction manifold in the case $k=3$, so the space under consideration is $\operatorname{Link}\left(P_{1}, P_{2}, P_{3} ; N\right)$. For higher $k$, one constructs $\left(\begin{array}{l}k \\ 3\end{array}\right)$ manifolds in the manner described below. There are two types of intersections which form the obstruction manifold. The first is the intersections of $P_{j}$ with the generalized Whitney disks for the pair $\left(P_{h}, P_{i}\right)$, where $h, i, j$ are distinct. The second is the intersections of the generalized Whitney circles of the pair $\left(P_{h}, P_{i}\right)$ with that for $\left(P_{i}, P_{j}\right)$, again for $h, i, j$ distinct.

6.3.1 Intersection of the Whitney disk with the $P_{i}$ Let $S=\{(1,2,3),(2,3,1)$, $(3,1,2)\}$. Let $T_{1}=\{(s, t) \mid 0 \leq s \leq t \leq 1\}$. For $(h, i, j) \in S$ consider the maps $\Phi_{1,(h i) j}: P_{1} \times P_{2} \times P_{3} \times T \rightarrow N^{4}$ given by

$$
\Phi_{1,(h i) j}\left(x_{1}, x_{2}, x_{3}, s, t\right)=\left(f_{j}\left(x_{j}\right), p_{1} H_{h i}\left(x_{h}, x_{i}, s\right), H_{h i}\left(x_{h}, x_{i}, t\right)\right) .
$$

Let $T_{2}=\{(s, t) \mid 0 \leq t \leq s \leq 1\}$. For $(h, i, j) \in S$ consider the maps $\Phi_{2,(h i) j}: P_{1} \times$ $P_{2} \times P_{3} \times T \rightarrow N^{4}$ given by

$$
\Phi_{2,(h i) j}\left(x_{1}, x_{2}, x_{3}, s, t\right)=\left(H_{h i}\left(x_{h}, x_{i}, s\right), p_{2} H_{h i}\left(x_{h}, x_{i}, t\right), f_{j}\left(x_{j}\right)\right) .
$$

Definition 6.3 For $a=1,2$, define $X_{a,(h i) j}=\Phi_{a,(h i) j}^{-1}\left(\Delta_{N} \times \Delta_{N}\right)$.

Proposition 13 For $a=1,2$, if $\Phi_{a,(h i) j}$ and its restrictions to $\partial_{1}\left(P_{1} \times P_{2} \times P_{3} \times T_{a}\right)$ and $\partial_{2}\left(P_{1} \times P_{2} \times P_{3} \times T_{a}\right)$ are transverse to $\Delta_{N} \times \Delta_{N}$, then $X_{a,(h i) j}$ is a compact $\left(p_{1}+p_{2}+p_{3}+2-2 n\right)$-dimensional manifold with at most 2 -strata, and stable normal bundle $T N \times T N-T P_{1} \times T P_{2} \times T P_{3}$. 
Proof Transversality gives an isomorphism

$$
T X_{a,(h i) j} \oplus T N \oplus T N \rightarrow T P_{1} \oplus T P_{2} \oplus T P_{3} \oplus \epsilon^{2} .
$$

The boundary of $X_{a,(h i) j}$ naturally decomposes in to three parts according to whether $t=0, s=0$ or $t=s . \partial X_{a,(h i) j}=\partial_{t} X_{a,(h i) j} \cup \partial_{s} X_{a,(h i) j} \cup \partial_{t=s} X_{a,(h i) j}$. Also, $\partial_{t=s} X_{1,(h i) j}=\partial_{t=s} X_{2,(h i) j}$. The 2-stratum of $X_{a,(h i) j}$ consists of the points $\left(p_{1}, p_{2}, p_{3}, 0,0\right)$ such that $f_{1}\left(p_{1}\right)=f_{2}\left(p_{2}\right)=f_{3}\left(p_{3}\right)$, a compact $\left(p_{1}+p_{2}+p_{3}-2 n\right)-$ dimensional manifold we will call $T$.

6.3.2 Intersections of the Whitney circles For $(h, i, j) \in S$, consider the maps $\Phi_{h i j}: P_{1} \times P_{2} \times P_{3} \times I \times I \rightarrow N^{4}$ given by

$$
\Phi_{h i j}\left(p_{1}, p_{2}, p_{3}, s, t\right)=\left(H_{h i}\left(p_{h}, p_{i}, s\right), H_{i j}\left(p_{i}, p_{j}, t\right)\right) .
$$

Definition 6.4 $W_{h i j}=\Phi_{h i j}^{-1}\left(\Delta_{N} \times \Delta_{N}\right)$.

Proposition 14 Suppose $\Phi_{h i j}$ and its restrictions to $\partial_{1}\left(P_{1} \times P_{2} \times P_{3} \times I \times I\right)$ and $\partial_{2}\left(P_{1} \times P_{2} \times P_{3} \times I \times I\right)$ are transverse to $\Delta_{N} \times \Delta_{N}$. Then $W_{h i j}$ is a compact $\left(p_{1}+p_{2}+p_{3}+2-2 n\right)$-dimensional manifold with boundary, and stable normal bundle $T N \times T N-T P_{1} \times T P_{2} \times T P_{3}$.

Proof Transversality gives an isomorphism

$$
T W_{h i j} \oplus T N \oplus T N \rightarrow T P_{1} \oplus T P_{2} \oplus T P_{3} \oplus \epsilon^{2} .
$$

The boundary $\partial W_{h i j}$ occurs when either $s=0$ or $t=0$, and gives a decomposition $\partial W_{h i j}=\partial_{s} W_{h i j} \cup \partial_{t} W_{h i j}$. The 2-stratum of $W_{h i j}$ is the set $T$ described above.

6.3.3 Forming the obstruction manifold The following lemma tells us how the boundaries of the $W_{h i j}$ and $X_{a,(h i) j}$ fit together, and can be verified by noting that the equations which define these manifolds are the same when the appropriate value of $t$ or $s$ is zero.

Lemma 6.5 For $(h, i, j) \in S$, there are diffeomorphisms of manifolds with boundary $\partial_{t} W_{h i j} \cong \partial_{t} X_{2,(h i) j}, \partial_{s} W_{h i j} \cong \partial_{s} X_{1,(i j) h}$, and $\partial_{s=t} X_{1,(h i) j} \cong \partial_{s=t} X_{2,(h i) j}$. 
We define $\partial X_{(h i) j}=\partial_{s=t} X_{1,(h i) j}$, and arrange this into the following diagram:

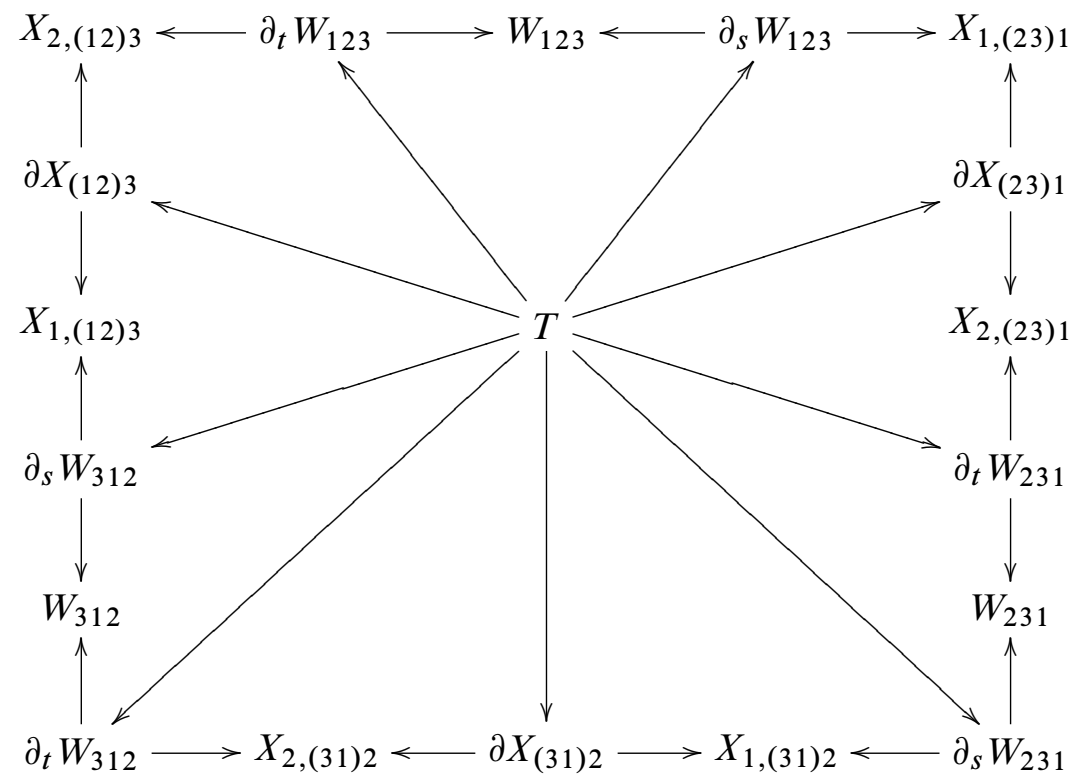

Let $\mathcal{I}$ denote the underlying category for this diagram, and let $\mathcal{Z}: \mathcal{I} \rightarrow$ Spaces be the functor which gives the diagram above.

Definition 6.6 Define $Z=\operatorname{colim}_{\mathcal{I}} \mathcal{Z}$, and let $Z^{\prime}=\operatorname{hocolim}_{\mathcal{I}} \mathcal{Z}$.

Note that the canonical map $a: Z^{\prime} \rightarrow Z$ is a homotopy equivalence. We wish to show how to piece together the bundle isomorphisms given by transversality in Proposition 13 and Proposition 14 to make a bundle isomorphism over $Z^{\prime}$. In order to do this, we need to compare the isomorphisms along the common boundary they define and invoke Proposition 4 and Proposition 5. In order to apply these propositions, we need to first describe the map $Z \rightarrow E_{123}$.

\subsection{The map $z: Z \rightarrow E_{123}$}

The map $z: Z \rightarrow E_{123}$ comes in several pieces, because $Z$ itself is defined in several pieces. Note that, by composition, this induces a map $z^{\prime}: Z^{\prime} \rightarrow E_{123}$. A point in $Z$ is a tuple $\left(x_{1}, x_{2}, x_{3}, s, t\right)$ which is in one of the $W_{h i j}$ or $X_{a,(h i) j}$. If $\left(x_{1}, x_{2}, x_{3}, s, t\right) \in$ $W_{h i j}$, then the corresponding point in $E_{123}$ has

$$
\omega_{h}\left(r_{h}\right)= \begin{cases}p_{1} H_{h i}\left(x_{h}, x_{i}, 2 r_{h} s\right) & \text { if } 0 \leq r_{h} \leq 1 / 2 \\ p_{2} H_{h i}\left(x_{h}, x_{i}, 2\left(1-r_{h}\right) s\right) & \text { if } 1 / 2 \leq r_{h} \leq 1,\end{cases}
$$


$\omega_{i}\left(r_{i}\right)=f_{i}\left(x_{i}\right)$, and

$$
\omega_{j}\left(r_{j}\right)= \begin{cases}p_{2} H_{i j}\left(x_{i}, x_{j}, 2 r_{j} t\right) & \text { if } 0 \leq r_{j} \leq 1 / 2 \\ p_{1} H_{i j}\left(x_{i}, x_{j}, 2\left(1-r_{j}\right) t\right) & \text { if } 1 / 2 \leq r_{j} \leq 1,\end{cases}
$$

for all $r_{i} \in[0,1]$. If $\left(x_{1}, x_{2}, x_{3}, s, t\right) \in X_{1,(h i) j}$, then

$$
\begin{aligned}
& \omega_{h}\left(r_{h}\right)=p_{1} H_{h i}\left(x_{h}, x_{i}, r_{h} s\right), \\
& \omega_{i}\left(r_{i}\right)= \begin{cases}p_{2} H_{h i}\left(x_{h}, x_{i}, 2 r_{i} t\right) & \text { if } 0 \leq r_{i} \leq 1 / 2 \\
p_{1} H_{h i}\left(x_{h}, x_{i}, 2\left(1-r_{i}\right) t+2\left(r_{i}-1 / 2\right) s\right) & \text { if } 1 / 2 \leq r_{j} \leq 1,\end{cases}
\end{aligned}
$$

and $w_{j}\left(r_{j}\right)=f_{j}\left(x_{j}\right)$ for all $r_{j} \in[0,1]$. Finally, if $\left(x_{1}, x_{2}, x_{3}, s, t\right) \in X_{2,(h i) j}$, then

$$
\omega_{h}\left(r_{h}\right)= \begin{cases}p_{1} H_{h i}\left(x_{h}, x_{i}, 2 r_{h} s\right) & \text { if } 0 \leq r_{j} \leq 1 / 2 \\ p_{2} H_{h i}\left(x_{h}, x_{i}, 2\left(1-r_{h}\right) s+2\left(r_{h}-1 / 2\right) t\right) & \text { if } 1 / 2 \leq r_{j} \leq 1,\end{cases}
$$

$\omega_{i}\left(r_{i}\right)=p_{2} H_{h i}\left(x_{h}, x_{i}, r_{i} t\right)$, and $\omega_{j}\left(r_{j}\right)=f_{j}\left(x_{i}\right)$ for all $r_{j} \in[0,1]$. By inspection, these maps agree on the intersections of the $W_{h i j}$ with the $X_{a,(h i) j}$. Hence we have a map $Z \rightarrow E_{123}$. Now we are going to produce a path in $C_{3}\left(P_{1}, P_{2}, P_{3} ; N\right)$ from an element $\alpha$ of

$$
\operatorname{hofiber}\left(\operatorname{Link}\left(P_{1}, P_{2}, P_{3} ; N\right) \rightarrow \mathcal{T}_{2} \operatorname{Link}\left(P_{1}, P_{2}, P_{3} ; N\right)\right) \text {. }
$$

Write $\alpha(u)=\left(H_{12}^{\prime}(u), H_{23}^{\prime}(u), H_{31}^{\prime}(u)\right)$, where $u \in I, \alpha(0)=\left(H_{12}, H_{23}, H_{31}\right) \in$ $\mathcal{T}_{2} \operatorname{Link}\left(P_{1}, P_{2}, P_{3} ; N\right)$, and $\alpha(1)=\left(H_{12}^{\prime}, H_{23}^{\prime}, H_{31}^{\prime}\right)$. Here $H_{i j}^{\prime}: P_{i} \times P_{j} \times I \rightarrow N \times N$ satisfies $H_{i j}^{\prime}=f_{i}^{\prime} \times f_{j}^{\prime}$ for some $\left(f_{1}^{\prime}, f_{2}^{\prime}, f_{3}^{\prime}\right) \in \operatorname{Link}\left(P_{1}, P_{2}, P_{3} ; N\right)$. Since we can think of $\alpha$ as a parametrized family of maps in $\mathcal{T}_{2} \operatorname{Link}\left(P_{1}, P_{2}, P_{3} ; N\right)$, we can apply the construction of $Z$ to this family. What remains is to give the map to $E_{123}$, which means associating three paths with the point. For the sake of brevity, let us do this in the specific case where $\left(x_{1}, x_{1}, x_{3}, s, t, u\right) \in W_{123}$. This means that $H_{12}\left(x_{1}, x_{2}, s, u\right) \in \Delta_{N}$ and $H_{23}\left(x_{2}, x_{3}, t, u\right) \in \Delta_{N}$. Then, for instance, we define

$$
\omega_{1}\left(r_{1}\right)= \begin{cases}p_{1} H_{12}\left(x_{1}, x_{2}, 2 r_{1} s, 2 r_{1} u\right) & \text { if } 0 \leq r_{j} \leq 1 / 2 \\ p_{2} H_{12}\left(x_{1}, x_{2}, 2\left(1-r_{1}\right) s, 2\left(1-r_{1}\right) u\right) & \text { if } 1 / 2 \leq r_{j} \leq 1 .\end{cases}
$$

The others are defined similarly.

\subsection{Gluing the bundle data}

For vector spaces $V, W$, let $L_{\max }(V, W)$ denote the space of linear maps $V \rightarrow W$ of maximal rank. There is an isomorphism $L_{\max }(V, W) \rightarrow L_{\max }(W, V)$ given by sending a linear transformation to its transpose. When $\operatorname{dim}(V) \leq \operatorname{dim}(W)$, the space $L_{\max }(V, W)$ is the Stiefel manifold, which is well-known to be 
$(\operatorname{dim}(W)-\operatorname{dim}(V)-1)-$ connected. We consider bundles of spaces $L_{\max }(V, W)$, as introduced in Definition 2.3.

Lemma 6.7 The derivatives $D \Phi_{h i j}$ and $D \Phi_{a,(h i) j)}$ are homotopic where it makes sense to compare them.

Proof Consider the space $L_{\max }\left(T P \times \epsilon^{2}, T N \times T N\right)$ as a space over $\partial_{t} W_{h i j} \cong$ $\partial_{t} X_{2,(h i) j}$. The maps $D \Phi_{h i j}$ and $D \Phi_{2,(h i) j)}$ define sections of these bundles, and we wish to show they are homotopic.

Take an open cover $\mathcal{U}$ of $\partial_{t} W_{h i j}$ consisting of contractible open sets $U$ so the bundle $L_{\max }\left(T P \times \epsilon^{2}, T N \times T N\right)$ is trivial over each $U \in \mathcal{U}$. Now $L_{\max }\left(T P \times \epsilon^{2}, T N \times T N\right)$ is $\left(p_{1}+p_{2}+p_{3}-2 n+1\right)$-connected, and if $p_{1}+p_{2}+p_{3}+2-2 n \leq 0$, there is nothing to prove. Otherwise, since $\left(p_{1}+p_{2}+p_{3}-2 n+1\right) \geq 0$, two such maps are always homotopic. It follows that $D \Phi_{h i j}$ and $D \Phi_{2,(h i) j}$ are homotopic, by Remark 1 . The same proof shows that the restrictions of $D \Phi_{h i j}$ and $D \Phi_{1,(i j) h}$ and $D \Phi_{1,(i j) h}$ and $D \Phi_{2,(i j) h}$ are homotopic where it makes sense to compare them. As for the 2strata $T$, we must compare these chosen homotopies on $T$ and ensure that the composed homotopy is homotopic to a constant homotopy. If $p_{1}+p_{2}+p_{3}+2-2 n \leq 1$ there is nothing to prove, since the 2 -strata is empty. Otherwise $\left(p_{1}+p_{2}+p_{3}-2 n+1\right) \geq 1$ and the space $L_{\max }\left(T P \times \epsilon^{2}, T N \times T N\right)$ is $\geq 1$-connected.

Lemma 6.8 There is an isomorphism $T Z \oplus T N \oplus T N \rightarrow T P_{1} \oplus T P_{2} \oplus T P_{3} \oplus \epsilon^{2}$.

Proof Let $P=P_{1} \times P_{2} \times P_{3}$, and identify $v\left(\Delta_{N} \times \Delta_{N} \subset N^{4}\right)$ with $T N \times T N$. For $(h, i, j) \in S$, the derivatives $D \Phi_{h i j}: T P \times \epsilon^{2} \rightarrow T N^{4}$ give sections of the bundles $L_{\max }\left(T P \times \epsilon^{2}, T N \times T N\right)$ over $W_{h i j}$. The same is true of $D \Phi_{a,(h i) j}: T P \times \epsilon^{2} \rightarrow$ $T N \times T N$ and the manifolds $X_{a,(h i) j}$. These maps are homotopic by Lemma 6.7. By Propositions 4 and 5, they combine to give an element of the space $\operatorname{hocolim}_{\mathcal{I}} L_{\max }\left(z^{\prime *} T P \times \epsilon^{2}, z^{\prime *} T N \times T N\right)$, which is a space over $Z^{\prime}$. Pulling back by a homotopy inverse to $a$ : $Z^{\prime} \rightarrow Z$, we obtain $\Phi \in L_{\max }\left(z^{*} T P \times \epsilon^{2}, z^{*} T N \times T N\right)$. The fiberwise kernel of $\Phi$ is, by construction, $T Z$ (see Proposition 2), and this gives the desired isomorphism.

Theorem 6.9 $Z$ can be given the structure of a smooth closed compact manifold of dimension $p_{1}+p_{2}+p_{3}+2-2 n$, and $T Z$ is stably isomorphic to $T P_{1} \times T P_{2} \times$ $T P_{3}-T N \times T N$.

Proof That $Z$ is smooth follows from Proposition 2, since the diffeomorphisms between the 1 -strata of the pieces which form $Z$ all restrict to the identity on $T$. Note 
that the canonical map $a: Z^{\prime} \rightarrow Z$ is a homotopy equivalence. By Lemma 6.8 , we pull back the bundle isomorphism over $Z^{\prime}$ by a homotopy inverse to $z$ to give the desired bundle isomorphism.

What we have shown is that there is a map from the subcomplex of 0 -simplices of

$$
\operatorname{hofiber}\left(\operatorname{Link}\left(P_{1}, P_{2}, P_{3} ; N\right) \rightarrow \mathcal{T}_{2} \operatorname{Link}\left(P_{1}, P_{2}, P_{3} ; N\right)\right)
$$

such that the above transversality conditions are met, to the 0 -simplices of the space $\Omega C_{3}\left(P_{1}, P_{2}, P_{3} ; N\right)$. Getting a map of simplicial sets is easy, since the above works for families of maps too, and since the subcomplex of transverse maps is homotopy equivalent to the full complex. Thus we have proven Theorem 1.3, restated below.

Theorem 6.10 For a basepoint in the image of $\operatorname{Link}\left(P_{1}, P_{2}, P_{3} ; N\right)$, there is a map of spaces

$l_{3}: \operatorname{hofiber}\left(\operatorname{Link}\left(P_{1}, P_{2}, P_{3} ; N\right) \rightarrow \mathcal{T}_{2} \operatorname{Link}\left(P_{1}, P_{2}, P_{3} ; N\right)\right) \rightarrow \Omega C_{3}\left(P_{1}, P_{2}, P_{3} ; N\right)$.

It would be interesting to know the connectivity of the map $l_{3}$.

\subsection{Example: The Borromean rings}

As an example, we show that the Borromean rings are not homotopic to the unlink. We proceed by finding a path from image of the Borromean rings to the unlink in $\mathcal{T}_{1} \operatorname{Link}\left(S^{1}, S^{1}, S^{1} ; \mathbf{R}^{3}\right)$, lifting this path to a path in $\mathcal{T}_{2} \operatorname{Link}\left(S^{1}, S^{1}, S^{1} ; \mathbf{R}^{3}\right)$, and then constructing the obstruction manifold for this family, which turns out to be a single point.

We need a lemma that ensures this construction really distinguishes the Borromean rings from the unlink, and it concerns the connecting map in the fibration sequence $\mathcal{L}_{3} \operatorname{Link}\left(S^{1}, S^{1}, S^{1} ; \mathbf{R}^{3}\right) \rightarrow \mathcal{T}_{3} \operatorname{Link}\left(S^{1}, S^{1}, S^{1} ; \mathbf{R}^{3}\right) \rightarrow \mathcal{T}_{2} \operatorname{Link}\left(S^{1}, S^{1}, S^{1} ; \mathbf{R}^{3}\right)$.

Lemma 6.11 $\pi_{1} \mathcal{T}_{2} \operatorname{Link}\left(S^{1}, S^{1}, S^{1} ; \mathbf{R}^{3}\right)$ acts trivially on $\pi_{0} \mathcal{L}_{3} \operatorname{Link}\left(S^{1}, S^{1}, S^{1} ; \mathbf{R}^{3}\right)$.

Proof Let $\mathcal{T}_{2}=\mathcal{T}_{2} \operatorname{Link}\left(S^{1}, S^{1}, S^{1} ; \mathbf{R}^{3}\right), \mathcal{L}_{3}=\mathcal{L}_{3} \operatorname{Link}\left(S^{1}, S^{1}, S^{1} ; \mathbf{R}^{3}\right), S^{(3)}=$ $S^{1} \times S^{1} \times S^{1}, \Phi_{3}=\operatorname{tfiber}\left(S \mapsto \operatorname{Emb}\left(S, \mathbf{R}^{3}\right)\right)$, and $\widehat{\Phi}_{3}=\operatorname{holim}_{S} \operatorname{Emb}\left(S, \mathbf{R}^{3}\right)$, where $S$ ranges over proper subsets of $\{1,2,3\}$.

Consider the following commutative diagram.

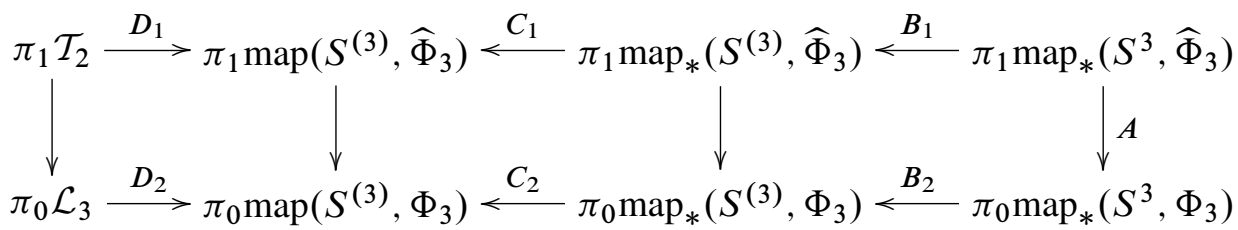


We wish to show the left-most vertical map is zero. It is enough to show that $A$ is the zero map, that $B_{1}$ and $B_{2}$ are injective, that $C_{1}$ and $C_{2}$ are isomorphisms, that $D_{2}$ is an isomorphism, and that the images $D_{1}$ and $C_{1} \circ B_{1}$ coincide.

That $D_{2}$ is an isomorphism follows from Theorem 4.5 by inspection, as the underlying spaces are the same. $C_{2}$ is an isomorphism since $\Phi_{3}$ is simply connected. $C_{1}$ is an isomorphism because we may regard $\pi_{1} \operatorname{map}_{*}\left(S^{(3)}, \widehat{\Phi}_{3}\right)=\pi_{0} \operatorname{map}_{*}\left(S^{(3)}, \Omega \widehat{\Phi}_{3}\right)$, and since $\Omega \widehat{\Phi}_{3}$ is an $H$-space, its fundamental group acts trivially on the based mapping space. For $i=1,2, B_{i}$ is induced by the cofiber sequence $X \rightarrow S^{(3)} \rightarrow S^{3}$, where $X=S^{1} \times S^{1} \times * \cup S^{1} \times * \times S^{1} \cup * \times S^{1} \times S^{1}$. If we suspend this cofiber sequence, we see that the map $\Sigma X \rightarrow \Sigma S^{(3)}$ has a section since $\Sigma X$ is an $H$-cospace. It follows from the long exact sequence in homotopy obtained by applying the functor map $_{*}\left(-, \Phi_{3}\right)$ that $B_{2}$ is injective. The same argument also shows that $B_{1}$ is injective.

To show that $A$ is the zero map, consider the following fibration sequence $\Phi_{3} \rightarrow$ $\operatorname{Emb}\left(\underline{3}, \mathbf{R}^{3}\right) \rightarrow \widehat{\Phi}_{3}$. We claim it has connecting map zero in homotopy. Consider the following diagram.

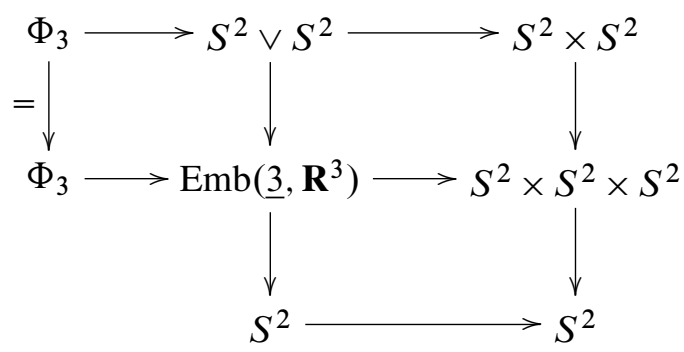

Everything in sight is a fibration sequence. The connecting map in the long exact sequence in homotopy of the top row is zero because, for spaces $X$ and $Y$, the map $\Omega(X \vee Y) \rightarrow \Omega(X \times Y)$ has a section. It follows that the connecting map for in the long exact sequence in homotopy of the middle row is zero as well.

Now consider the map of spaces $\mathcal{T}_{2} \rightarrow \operatorname{map}\left(S^{(3)}, \widehat{\Phi}_{3}\right)$ which induces $D_{1}$. Note that $\widehat{\Phi}_{3} \simeq \prod_{3} S^{2}, \mathcal{T}_{2}=\prod_{3} \operatorname{map}\left(S^{1} \times S^{1}, S^{2}\right)$, and the map which induces $D_{1}$ is compatible with these equivalences and may therefore be interpreted as the map $\prod_{3} \operatorname{map}\left(S^{1} \times S^{1}, S^{2}\right) \rightarrow \prod_{3} \operatorname{map}\left(S^{(3)}, S^{2}\right)$ which sends a triple $(f, g, h)$ to the map $F(x, y, z)=(f(x, y), g(y, z), h(z, x))$. On the one hand, the component of $\prod_{3} \operatorname{map}\left(S^{1} \times S^{1}, S^{2}\right)$ in which we choose the basepoint is the degree zero maps (the image of the unlink is the basepoint), and thus consists of those maps $F=$ $(f, g, h)$ such that the components are independent of one of the three variables and are null homotopic. On the other hand, $\operatorname{map}_{*}\left(S^{3}, \widehat{\Phi}_{3}\right)$ is the fiber of the fibration $\operatorname{map}_{*}\left(S^{(3)}, \widehat{\Phi}_{3}\right) \rightarrow \operatorname{map}_{*}\left(X, \widehat{\Phi}_{3}\right)$, and thus consists of those maps $S^{(3)} \rightarrow \widehat{\Phi}_{3}$ which 
are constant on $X$. It is homotopy equivalent to the homotopy fiber of this map, which consists of those maps $S^{(3)} \rightarrow \widehat{\Phi}_{3}$ which are homotopic to a map constant on $X$. This is precisely the image of $\mathcal{T}_{2}$ in $\prod_{3} \operatorname{map}\left(S^{(3)}, S^{2}\right)$.

Let $B=\left(f_{1}, f_{2}, f_{3}\right) \in \operatorname{Link}\left(S^{1}, S^{1}, S^{1} ; \mathbf{R}^{3}\right)$ be the Borromean rings, where $f_{1}(x)=$ $(0,2 \cos x, \sin x), f_{2}(y)=(\cos y, 0,2 \sin y)$, and $f_{3}(z)=(2 \cos z, \sin z, 0)$. Let the unlink be represented by the triple $U=\left(f_{1}, e_{2}, f_{3}\right) \in \operatorname{Link}\left(S^{1}, S^{1}, S^{1} ; \mathbf{R}^{3}\right)$, where $e_{2}(y)=(\cos y, 4,2 \sin y)$. The images of $B$ and $U$ in $\mathcal{T}_{1} \operatorname{Link}\left(S^{1}, S^{1}, S^{1} ; \mathbf{R}^{3}\right)$ are clearly homotopic by the path $L(t)=\left(f_{1}, H_{1}(y, t), f_{3}\right)$, where $H_{1}(y, t)=$ $(\cos y, t, 2 \sin y)$ for $t \in[0,4]$. We lift this path to the path in $\mathcal{T}_{2} \operatorname{Link}\left(S^{1}, S^{1}, S^{1} ; \mathbf{R}^{3}\right)$ given by the triple $\left(f_{1} \times f_{3}, H_{1} \times f_{1}, f_{3} \times H_{3}\right)$, where $H_{3}(y, t)$ is given by $\left(\cos y, t, 2 \sin y-(t-2)^{2}+4\right)$ for $t \in[0,4]$ (we have altered the order in which we represent tuples in $\mathcal{T}_{2}$ that we used in Section 6.1, and the $\mathcal{T}_{2}$ we mean here is the image of an inner automorphism of $\mathcal{T}_{2}$ induced by a permutation of $\{1,2,3\}$ ).

In order to construct the obstruction from this family, we must make the double point construction, and form the Whitney disks. The double points of the family $L(t)$ come from the intersection of $f_{3}$ with $H_{1}$, which is clearly a pair of points $(y, z, t)=$ $(\pi / 2, \pi / 3, \sqrt{3} / 2),(3 \pi / 2,2 \pi / 3, \sqrt{3} / 2)$, and this intersection is transverse. To make a nullcobordism of this manifold, we define a function $G: S^{1} \times S^{1} \times[0,4] \times[0,1] \rightarrow$ $\mathbf{R}^{3} \times \mathbf{R}^{3}$ given by $G(y, z, t, s)=\left(f_{3}(z), s H_{3}(y, t)+(1-s) H_{1}(y, t)\right)$.

Proposition $15 G$ is transverse to $\Delta_{\mathbf{R}^{3}}$ and $G^{-1}\left(\Delta_{\mathbf{R}^{3}}\right) \subset S^{1} \times S^{1} \times[0,4] \times[0,1]$ is an arc which embeds in each copy of $S^{1}$ under the projections to the $S^{1}$ factors.

Proof Let $H(y, t, s)=s H_{3}(y, t)+(1-s) H_{1}(y, t)$. To see that $G$ is transverse to the diagonal, it is equivalent to check that the images of $f_{3}$ and $H$ intersect transversely in $\mathbf{R}^{3}$. The derivative

$$
D H=\left(\begin{array}{ccc}
-\sin y & 0 & 0 \\
0 & 1 & 0 \\
2 \cos y & -2 s(t-2) & -(t-2)^{2}+4
\end{array}\right)
$$

has determinant $\left(-(t-2)^{2}+4\right) \sin y$, which vanishes if and only if either $t=0,4$ or $y=0, \pi$. Since there are no solutions to $f_{3}(z)=H(y, 0, s)$, we only have to deal with the possibility of $y=0, \pi$. In this case, $z=\pi / 3,2 \pi / 3$, and by inspection we find that for these values of $z, D f_{3}$ and the last two columns of $D H$ span $\mathbf{R}^{3}$.

From transversality it follows that $D=G^{-1}\left(\Delta_{\mathbf{R}^{3}}\right)$ is a 1-dimensional manifold with boundary. It is the solutions to the following equations:

$$
2 \cos z=\cos y, \quad \sin z=t, \quad 0=2 \sin y+s\left(-(t-2)^{2}+4\right)
$$


The first two equations imply that $z \in[\pi / 3,2 \pi / 3]$ and $t \in[\sqrt{3} / 2,1]$. Moreover, for each such $(z, t)$ there is a unique $(y, s)$ which solves all three, since the third equation implies that $2 \sin y \leq 0$, and we can solve for $s$. Let $p_{i}: S^{1} \times S^{1} \times[0,4] \times[0,1] \rightarrow S^{1}$ for $i=1,2$ be the projections. We have shown that $p_{2}(D)=[\pi / 3,2 \pi / 3]$ and that the restriction of $p_{2}$ to $D$ is an embedding. It is also clear that $p_{1}(D)=[\pi, 2 \pi]$ and that the projection $p$ of $D$ onto $S^{1} \times I$, given by $p(y, z, t)=(y, t)$, is an embedding (in fact, the restriction of $p_{1}$ itself is an embedding, though we will not need this).

The Whitney circle $C=f_{3}\left(p_{2} D\right) \cup H_{1}(p D)$ is a bent circle in $\mathbf{R}^{3}$. Let $\pi_{i}: \mathbf{R}^{3} \times \mathbf{R}^{3} \rightarrow$ $\mathbf{R}^{3}$ be the projections for $i=1,2$. $C$ bounds a disk $W$ given by $G$ as follows: For each $(y, z, t, s) \in D, G$ gives rise to a path in $\mathbf{R}^{3}$ from $f_{3}(z)=\pi_{1} G(y, z, t, 0)$ to $H_{1}(y, t)=\pi_{2} G(y, z, t, 0)$ because $\pi_{1} G(y, z, t, s)=\pi_{2} G(y, z, t, s)$. The path is defined by

$$
\gamma(\sigma)= \begin{cases}\pi_{1} G(y, z, t, 2 \sigma s) & \text { if } \sigma \in[0,1 / 2] \\ \pi_{2} G(y, z, t,(2-2 \sigma) s) & \text { if } \sigma \in[1 / 2,1] .\end{cases}
$$

The union of these paths forms the disk $W$ bounding $C$ in $\mathbf{R}^{3}$, and it is clearly disjoint from $f_{3}\left(S^{1}\right)$ and $H_{1}\left(S^{1}\right) \times[0,4]$ except along its boundary. It is clear that $f_{2}\left(S^{1}\right)$ intersects $W$ transversely in a single point, since $W$ is the union of straight lines between $z$ and $(y, t)$ for each $(y, z, t, s) \in D$ (and for each $y$ there is a unique $z$ and vice-versa, since the projections of $D$ to the $S^{1}$ factors are embeddings). It follows that $B$ cannot be link homotopic to $U$, since $\pi_{1} \mathcal{T}_{2} \operatorname{Link}\left(S^{1}, S^{1}, S^{1} ; \mathbf{R}^{3}\right)$ acts trivially on $\pi_{0} \mathcal{L}_{3} \operatorname{Link}\left(S^{1}, S^{1}, S^{1} ; \mathbf{R}^{3}\right)$ by Lemma 6.11 .

Acknowledgments The author would like to thank Greg Arone, Ryan Budney, Vladimir Chernov, Ralph Cohen, Tom Goodwillie and Steve Kerckhoff for helpful conversations. He would also like to thank Don Stanley, who first posed the question to the author about whether the linking number could be seen using calculus of functors. Finally he would like to thank the referee for helpful comments and supplying more references. This research was partly supported by NSF grant DMS-0402822.

\section{References}

[1] V V Chernov, Y B Rudyak, Toward a general theory of linking invariants, Geom. Topol. 9 (2005) 1881-1913 MR2175159

[2] T G Goodwillie, Calculus. I. The first derivative of pseudoisotopy theory, $K$-Theory 4 (1990) 1-27 MR1076523

[3] T G Goodwillie, J R Klein, Excision estimates for spaces of Poincaré embeddings, In preparation 
[4] T G Goodwillie, J R Klein, MS Weiss, Spaces of smooth embeddings, disjunction and surgery, from: "Surveys on surgery theory, Vol. 2", Ann. of Math. Stud. 149, Princeton Univ. Press (2001) 221-284 MR1818775

[5] T G Goodwillie, J R Klein, MS Weiss, A Haefliger style description of the embedding calculus tower, Topology 42 (2003) 509-524 MR1953238

[6] T G Goodwillie, B Munson, Connectivity estimates for spaces of link maps, In preparation

[7] T G Goodwillie, M Weiss, Embeddings from the point of view of immersion theory: Part II, Geom. Topol. 3 (1999) 103-118 MR1694808

[8] N Habegger, U Kaiser, Link homotopy in the 2-metastable range, Topology 37 (1998) 75-94 MR1480878

[9] N Habegger, X-S Lin, The classification of links up to link-homotopy, J. Amer. Math. Soc. 3 (1990) 389-419 MR1026062

[10] A Hatcher, F Quinn, Bordism invariants of intersections of submanifolds, Trans. Amer. Math. Soc. 200 (1974) 327-344 MR0353322

[11] M W Hirsch, Differential topology, Graduate Texts in Math. 33, Springer, New York (1976) MR0448362

[12] J Klein, BWilliams, Homotopical intersection theory I arXiv:math.AT/0512479

[13] U Koschorke, A generalization of Milnor's $\mu$-invariants to higher-dimensional link maps, Topology 36 (1997) 301-324 MR1415590

[14] U Koschorke, Link maps in arbitrary manifolds and their homotopy invariants, J. Knot Theory Ramifications 12 (2003) 79-104 MR1953625

[15] U Koschorke, Link homotopy in $S^{n} \times \mathbb{R}^{m-n}$ and higher order $\mu$-invariants, J. Knot Theory Ramifications 13 (2004) 917-938 MR2101235

[16] U Koschorke, Linking and coincidence invariants, Fund. Math. 184 (2004) 187-203 MR2128050

[17] U Koschorke, D Rolfsen, Higher-dimensional link operations and stable homotopy, Pacific J. Math. 139 (1989) 87-106 MR1010788

[18] J P Levine, An approach to homotopy classification of links, Trans. Amer. Math. Soc. 306 (1988) 361-387 MR927695

[19] W S Massey, D Rolfsen, Homotopy classification of higher-dimensional links, Indiana Univ. Math. J. 34 (1985) 375-391 MR783921

[20] J Milnor, Link groups, Ann. of Math. (2) 59 (1954) 177-195 MR0071020

[21] J Milnor, Topology from the differentiable viewpoint, University Press of Virginia, Charlottesville, VA (1965) MR0226651 Based on notes by D W Weaver

[22] B A Munson, Embeddings in the 3/4 range, Topology 44 (2005) 1133-1157 MR2168572 
[23] R Schneiderman, $\mathbf{P}$ Teichner, Higher order intersection numbers of 2-spheres in 4-manifolds, Algebr. Geom. Topol. 1 (2001) 1-29 MR1790501

[24] G P Scott, Homotopy links, Abh. Math. Sem. Univ. Hamburg 32 (1968) 186-190 MR0236912

[25] A Skopenkov, On the generalized Massey-Rolfsen invariant for link maps, Fund. Math. 165 (2000) 1-15 MR1804997

[26] M Weiss, Embeddings from the point of view of immersion theory: Part I, Geom. Topol. 3 (1999) 67-101 MR1694812

Department of Mathematics, Wellesley College

Wellesley, MA 02481, USA

bmunson@wellesley.edu

http://palmer.wellesley.edu/ munson

Received: 16 April $2008 \quad$ Revised: 30 October 2008 